\title{
Mining the gap: evolution of the magnitude gap in X-ray galaxy groups from the 3-square-degree XMM coverage of CFHTLS ${ }^{\star}$
}

\author{
G. Gozalias ${ }^{1,2,3}$, A. Finoguenov ${ }^{1}$, H. G. Khosroshahi ${ }^{4}$, M. Mirkazemi ${ }^{5}$, M. Salvato ${ }^{5}$, D. M. Z. Jassur ${ }^{2}$, G. Erfanianfar ${ }^{5}$, \\ P. Popesso ${ }^{11}$, M. Tanaka ${ }^{6}$, M. Lerchster ${ }^{5}$, J. P. Kneib ${ }^{7}$, H. J. McCracken ${ }^{8}$, Y. Mellier ${ }^{8}$, E. Egami ${ }^{9}$, M. J. Pereira ${ }^{9}$, \\ F. Brimioulle ${ }^{5}$, T. Erben ${ }^{10}$, and S. Seitz ${ }^{5}$
}

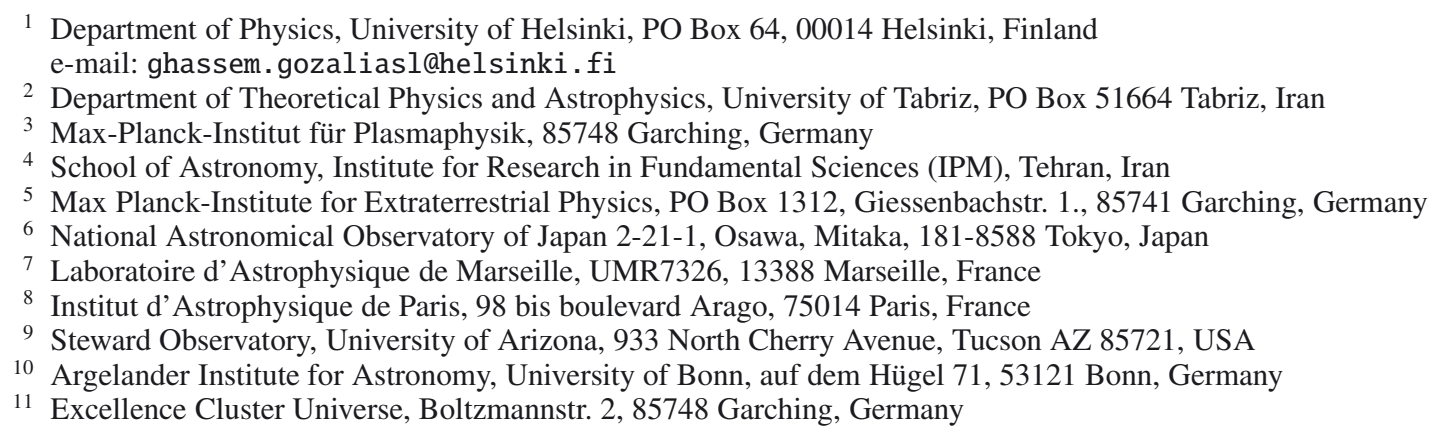

Received 7 August 2013 / Accepted 13 May 2014

\section{ABSTRACT}

\begin{abstract}
We present a catalog of $129 \mathrm{X}$-ray galaxy groups, covering a redshift range $0.04<z<1.23$, selected in the $\sim 3 \mathrm{deg}^{2}$ part of the CFHTLS W1 field overlapping XMM observations performed under the XMM-LSS project. We carry out a statistical study of the redshift evolution out to redshift one of the magnitude gap between the first and the second brightest cluster galaxies of a well defined mass-selected group sample. We find that the slope of the relation between the fraction of groups and the magnitude gap steepens with redshift, indicating a larger fraction of fossil groups at lower redshifts. We find that $22.2 \pm 6 \%$ of our groups at $z \leq 0.6$ are fossil groups. We compare our results with the predictions of three semi-analytic models based on the Millennium simulation. The intercept of the relation between the magnitude of the brightest galaxy and the value of magnitude gap becomes brighter with increasing redshift. This trend is steeper than the model predictions which we attribute to the younger stellar age of the observed brightest cluster galaxies. This trend argues in favor of stronger evolution of the feedback from active galactic nuclei at $z<1$ compared to the models. The slope of the relation between the magnitude of the brightest cluster galaxy and the value of the gap does not evolve with redshift and is well reproduced by the models, indicating that the tidal galaxy stripping, put forward as an explanation of the occurrence of the magnitude gap, is both a dominant mechanism and sufficiently well modeled.
\end{abstract}

Key words. methods: statistical - techniques: photometric - Galaxy: evolution - galaxies: clusters: general $\mathrm{X}$-rays: galaxies: clusters - surveys

\section{Introduction}

Groups and clusters are important environments for the formation and evolution of galaxies, particularly for the very bright galaxies. In the last few decades, several observational and theoretical studies have focused on the group and cluster environments in order to advance our understanding of their galaxy properties (e.g., White \& Rees 1978; Yang et al. 2005, 2008, 2009; Zandivarez et al. 2006; Zibetti et al. 2009; Percival et al. 2010; van den Bosch et al. 2013; Hearin et al. 2013; Scoville et al. 2007).

While effects of gravity on galaxy formation have been modeled in great detail (e.g., Zel'dovich 1970; Weinberg 1972; Press \& Schechter 1974; Huntley 1980; Barnes 1989; Bekki et al. 2002; Libeskind et al. 2006; Junqueira et al. 2013), processes of the galaxy evolution are also governed by the gas content

\footnotetext{
$\star$ Appendix $\mathrm{A}$ is available in electronic form at http://www . aanda.org
}

available for star-formation. For galaxies in groups and clusters, the cold gas content depends on both external (cooling of the intracluster medium (ICM), gas stripping by galactic motion through ICM, observed even for the central galaxy due to sloshing), and internal processes galactic outflows, driven by the feedback from active galactic nuclei (AGN) and supernovae (Croton et al. 2006). In addition, the central galaxy can grow by merging and tidal stripping of satellite galaxies.

Dynamical friction causes massive galaxies in halos with sufficiently low velocity dispersion to merge in a few Gyr with the central group galaxy, forming a giant elliptical galaxy (Ponman et al. 1994). Within this galaxy-galaxy merger paradigm, luminosity gap may be sensitive to merger rates in groups and clusters of galaxies. To get the insights on the processes associated with the formation of central galaxies, we embark on a study of the occurrence of the magnitude gap.

The magnitude gap is often used to study the formation history and dynamical age of the hosting halo, particularly in fossil 
groups (Milosavljević et al. 2006; van den Bosch et al. 2007; Dariush et al. 2007). Simulations of D'Onghia et al. (2005) show that early formed groups (fossils) have larger magnitude gaps. Tracing fossil groups forward in time, Von Benda-Beckmann et al. (2008) find that their large magnitude gaps can be filled by a recent infall of galaxies. Also, some merger events may not be caught by the gap statistics, as indicated by a study of La Barbera et al. (2012). Thus, to derive conclusions from the gap statistics, a comparison to numerical simulations is required. To date, such a comparison has only been performed on the nearby galaxy groups at $z \sim 0$ and massive clusters at $z \sim 0.2$ (Dariush et al. 2010; Smith et al. 2010). This paper expands the studies of the cosmic evolution of the magnitude gap to a redshift of 1.1.

To the extremes of the magnitude gap characterization belong the fossil groups, which have $L_{\mathrm{X}} \gtrsim 2.13 \times 10^{42} \mathrm{~h}^{-2} \mathrm{erg} \mathrm{s}^{-1}$ and a magnitude gap above $\Delta M_{1,2}=2 \mathrm{mag}$ (Jones et al. 2003). Origin and nature of fossil groups are still not completely understood. Identification of these systems is challenging, and further studies and observations (i.e., X-ray and spectroscopic) of fossil groups are of interest. According to Jones et al. (2003), 8 to 20 per cent of all galaxy groups are fossils. Simulations suggest that fossils tend to reside in less dense environments compared to an average group of similar total mass and formation history (D’Onghia et al. 2005; Dariush et al. 2007; Díaz-Giménez et al. 2011). This is also observed in the environmental study of a fossil group, ESO 3060170 ( $\mathrm{Su}$ et al. 2013). Fossil galaxy groups have been shown to exhibit interesting properties such as concentrated dark matter halos (Khosroshahi et al. 2007; Humphrey et al. 2012), disky isophotes of the central galaxies (Khosroshahi et al. 2006; Smith et al. 2010). From the age and metallicity gradients of fossils, in a study based on six galaxies Eigenthaler \& Zeilinger (2013) concluded that these systems form by multiple mergers of massive galaxies.

Identification and detection of galaxy groups is also challenging, since the overdensity of galaxies per unit area in a redshift slice is more sensitive to the projection effects by foreground and background galaxies compared to clusters. In addition, to study the galaxy properties it is advantageous to have a group selection that is independent of galaxy properties. Extended X-ray emission from groups and clusters of galaxies offers such a selection (Borgani \& Guzzo 2001; Rosati et al. 2002). Deep XMM and Chandra surveys such as Chandra Deep Field South (CDFS; Giacconi et al. 2002), Chandra Deep Field North (CDFN; Bauer et al. 2002), Lockman Hole (Finoguenov et al. 2005), the Cosmic Evolution Survey (COSMOS; Finoguenov et al. 2007), XMM-Large Scale Structure (LSS; Pacaud et al. 2007), the Canadian Network for Observational Cosmology (CNOC2; Finoguenov et al. 2009; Connelly et al. 2012), Subaru-XMM Deep Field (SXDF; Finoguenov et al. 2010), the XMM-Newton-Blanco Cosmology Survey project (XMM-BCS; Šuhada et al. 2012) reveal new capabilities of X-ray selection of groups. Catalogs available from these surveys have already made an important contribution to studies of galaxy formation and evolution (e.g., Tanaka et al. 2008, 2012; Giodini et al. 2009; Silverman et al. 2009; Allevato et al. 2012).

The main goal of this paper is to identify X-ray galaxy groups in the part of the CFHTLS W1 field covered by XMM observations. We carry out a statistical study of the redshift evolution, up to a redshift of 1.1, of the magnitude gap and the volume abundance of the detected X-ray galaxy groups. We combine the observational measurements of the distribution of the luminosity gap of groups and the absolute magnitude of the brightest galaxies, and compare this to predictions of semi-analytic models
(SAMs) from Bower et al. (2006, hereafter B06), De Lucia \& Blaizot (2007, hereafter DLB07) and Guo et al. (2011, hereafter G11). The study of magnitude gap statistics and X-ray properties of galaxy groups enable us to identify fossil group candidates in our sample. We also report on the redshift evolution of the extent of X-ray detection from galaxy groups.

The structure of this paper is as follows: we present the XMM-Newton and CFHTLS data in Sect. 2. Section 3 gives a brief description of the SAMs we use in this paper, highlighting their differences and similarities. In Sect. 4, we describe the group identification technique and present a catalog of galaxy groups. Section 5 discusses group membership contamination. We present magnitude gap statistics and relation between the magnitude of the first and second brightest group galaxies (BGGs) and the magnitude gap in Sect. 6. We summarize our results in Sect. 7.

Unless stated otherwise, we adopt a "concordance" cosmological model, with $\left(\Omega_{\Lambda}, \Omega_{\mathrm{M}}, h\right)=(0.75,0.25,0.71)$, where the Hubble constant is characterized as $100 \mathrm{~h} \mathrm{~km} \mathrm{~s}^{-1} \mathrm{Mpc}^{-1}$ and quote uncertainties on $68 \%$ confidence level.

\section{Data}

\subsection{XMM-Newton data}

A description of the XMM-Newton observatory is given by Jansen et al. (2001). We use the data collected by the European photon imaging cameras (EPIC): the pn-CCD camera (Strüder et al. 2001) and the MOS-CCD cameras (Turner et al. 2001).

In this paper we analysed the XMM-Newton observations of the CFHTLS wide (W1) field as a part of the XMM-LSS survey (Pierre et al. 2007). The details of observations and data reduction are presented in Bielby et al. (2010). We concentrate on the low- $z$ counterparts of the X-ray sources and use all XMM observations performed till 2009 , covering an area of $2.276^{\circ} \times 2.276^{\circ}$. Altogether, $\sim 3$ degrees $^{2}$ of this area are covered by the optical data of the CFHTLS survey. The primary cluster catalog of the XMM-LSS project is published in Adami et al. (2011). The group catalog presented in this paper extends the Adami et al. (2011) work to low-mass systems, as well as presents X-ray flux estimates with contribution from point sources removed. This allows us to self-consistently infer cluster masses, using the calibrations achieved in the COSMOS survey using a similar flux extraction technique (Leauthaud et al. 2010). In our modeling of the survey sensitivity, we account for both the variation in the exposure due to differences in the flare removal, vignetting, and the aperture flux loss for nearby groups.

The modeling of the flux limit is defined by the spatial scales used for the detection and the sensitivities achieved on these scales. The change in the flux limit with redshift occurs at redshifts below 0.2 and is due to the correction for the missing flux, while the aperture flux limit is considered to be redshift independent. The correction for the missing flux is done following the tabulations of Finoguenov et al. (2007).

In Fig. 1 we present the flux limit corresponding to the best $10 \%$ (solid red curve) and 90\% (dotted blue curve) of the area. In addition to an increase in the flux, very low- $z$ systems are hard to identify in the photometric data due to the projection effects. The volume covered at low- $z$ (e.g., $z<0.05$ ) is very small, $0.02 \%$ of the total survey volume, while formal galaxy group luminosities are below $10^{41} \mathrm{ergs} \mathrm{s}^{-1}$ level, beyond the explored level for galaxy groups. The dashed black horizontal line in Fig. 1 illustrates the $10^{-14} \mathrm{ergs} \mathrm{s}^{-1} \mathrm{~cm}^{-2}$ flux threshold adopted for the $\mathrm{C} 1$ XMM-LSS sample (Pacaud et al. 2007). The open black squares 
G. Gozaliasl et al.: Evolution of the luminosity gap in X-ray galaxy groups

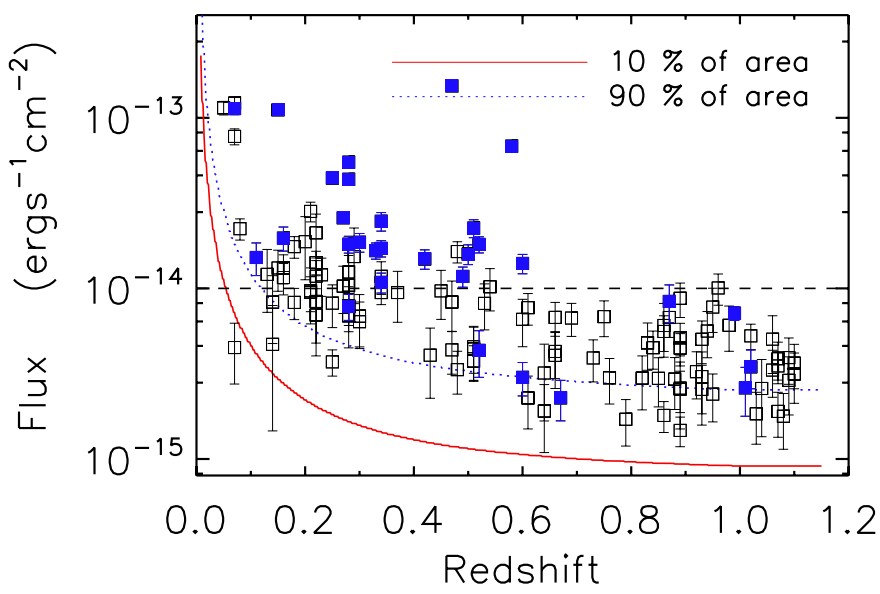

Fig. 1. Detected flux $(0.5-2 \mathrm{keV}$ band) as a function of redshift for the sample and its modeling. The open black squares with error bars present our identified galaxy groups. The filled blue squares mark the galaxy groups in common with Adami et al. (2011) catalog. Solid red (dotted blue) curve shows the sensitivity achieved at the deepest $10(90) \%$ area of the survey. The horizontal dashed black line shows the $10^{-14} \mathrm{ergs} \mathrm{s}^{-1} \mathrm{~cm}^{-2}$ flux threshold adopted for the C1 XMM-LSS sample.

with error bars show our flux (ergs s${ }^{-1} \mathrm{~cm}^{-2}, 0.5-2 \mathrm{keV}$ band) estimates versus redshift. We show 30 galaxy groups in common with Adami et al. (2011) with filled blue squares. These 30 galaxy groups include one system from $\mathrm{C} 0$, all 17 systems from $\mathrm{C} 1$ which are within the CFHTLS coverage, five systems from $\mathrm{C} 2$ and seven systems from C3 in the XMM-LSS classification. Using simulations, Pacaud et al. (2006) classified extended sources as $\mathrm{C} 1$ and $\mathrm{C} 2$. The $\mathrm{C} 1$ class is a purely $\mathrm{X}$-ray selected cluster (i.e., less than $1 \%$ of clusters can be found as miss-classified point-like source). The $\mathrm{C} 2$ class allows for the $50 \%$ contribution from the miss-classified point-liked sources. The C3 clusters are classified as faint objects with less-well characterized X-ray properties. These objects have been selected mostly by visual inspection of the optical and X-ray data. The $\mathrm{C} 0$ class considered as the low mass cluster category without clear X-ray emission. The detailed information is presented in Adami et al. (2011); Pacaud et al. (2006, 2007).

In Fig. 2 we compare our flux estimates in the $0.5-2 \mathrm{keV}$ band for the galaxy groups and clusters in common with Adami et al. (2011). As can be seen, our estimates of flux for these groups are generally consistent, but some groups, notoriously all the groups at $z>0.6$ (filled symbols in Fig. 2), have lower flux in our analysis. In Fig. 2, we also study the possibility of these differences to stem from the extrapolation of the flux, and find it unlikely, as the values for radii of $R_{500}$ we use are comparable to the $0.5 \mathrm{Mpc}$ radius, used in Adami et al. (2011). All these high- $z$ groups have strong contribution from point sources to the emission, which we detect and remove, which would explain the differences in the flux estimates. Most of these point sources are not aligned with cluster center and can hardly be associated with unresolved cool cores. A previous case of such disagreement in the flux, related to the $z=1.6$ cluster (compare Tanaka et al. 2010; Papovich et al. 2010), has been settled by Chandra observation in favor of our flux (Pierre et al. 2012). Our own correction for the removal of cool core flux of high- $z$ groups, based on the Chandra data on COSMOS, included in the analysis of Leauthaud et al. (2010), is $10 \%$ on average. This correction is applied when using the scaling relations.

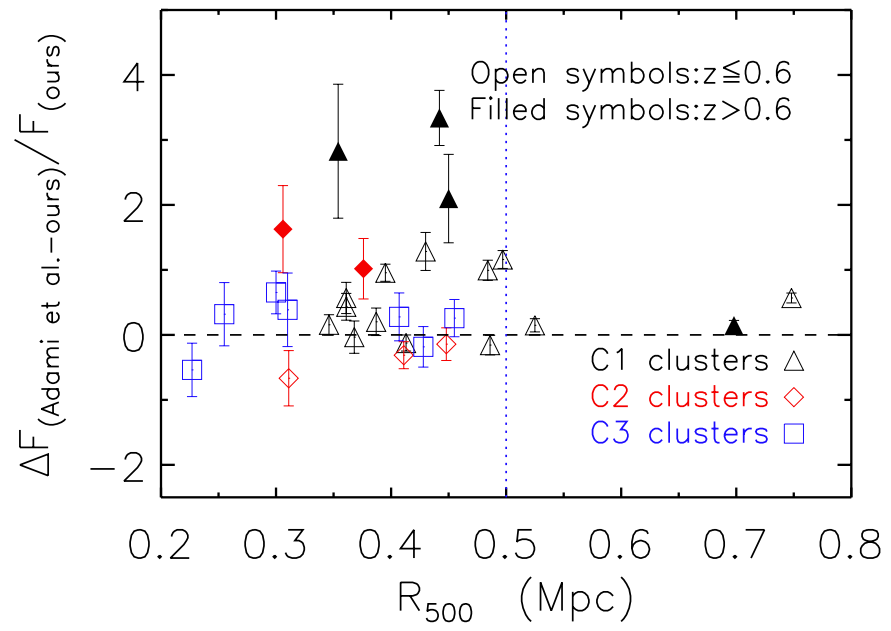

Fig. 2. Comparison of the reported flux in the $0.5-2 \mathrm{keV}$ band for galaxy groups and clusters in common between Adami et al. (2011) and our catalog, $\left(\right.$ Flux $_{\text {Adami }} /$ Flux $\left._{\text {Ours }}-1\right)$ versus $R_{500}(\mathrm{Mpc})$. We illustrate $\mathrm{C} 1$ clusters with black triangles, $\mathrm{C} 2$ clusters with red diamonds and $\mathrm{C} 3$ clusters with blue squares. The filled and open symbols show galaxy groups and clusters at redshift ranges $z>0.6$ and $z \leq 0.6$, respectively. The vertical dashed blue line at $0.5 \mathrm{Mpc}$, marks the fixed radius used for the flux estimates in Adami et al. (2011).

Our modeling of the survey volume, used for refined selection of the mock galaxy catalogs, takes into account the relation between the luminosities we extract from our flux estimates and a total mass, inferred by the weak lensing analysis (Leauthaud et al. 2010) on systems of similar mass and redshift found in the COSMOS field. The two-dimensional information on the sensitivity toward the flux detection and the resulting flux limits as a function of redshift discussed above.

\subsection{Photometric and spectroscopic data}

The CFHTLS wide observations have been carried out in the period between 2003 and 2008, covering an effective survey area of 154 square degrees. The W1 field is the widest field of the CFHTLS survey and covers an area of $\sim 64$ square degrees around $\mathrm{RA}=02^{\mathrm{h}} 18^{\mathrm{m}} 00^{\mathrm{s}}, \mathrm{Dec}=07^{\mathrm{h}} 00^{\mathrm{m}} 00^{\mathrm{s}}$. The optical images and data of the CFHTLS were obtained with the MegaPrime instrument mounted on the CFHT in the five filters $u^{*}, g^{\prime}, r^{\prime}$, $i^{\prime}$ and $z^{\prime}$ (e.g., Erben et al. 2013).

The completeness limits and image quality of the photometric survey data are important for the detection of the optical counterparts of the X-ray sources and for the accuracy of the photometric redshift of galaxies (e.g., Mirkazemi et al. 2014; Brimioulle et al. 2008; Bordoloi et al. 2012).

For the purposes of our study the completeness toward the detection of the second brightest galaxies is important for the large magnitude gap systems at high-z.

In Fig. 3 we show the completeness limits, $r^{\prime} \sim 25$ mag (vertical dotted black line), $i^{\prime} \sim 24.5 \mathrm{mag}$ and $z^{\prime} \sim 24 \mathrm{mag}$ for the optical data of CFHTLS-W1 field overlapping XMM observations. We also compare the $r^{\prime}$-band magnitude distribution of BGGs in our catalog in two redshift ranges, $z \leq 0.5$ (solid black histogram) and $z>0.5$ (dashed black histogram), to the $r^{\prime}$-band completeness limit of the CFHTLS.

This comparison indicates that completeness limit has no effect on the magnitude gap calculation of groups with $\Delta M_{1,2}<2$. However for the $z>0.5$ groups with $\Delta M_{1,2}>2$, the determination of the gap is affected by the completeness. In order to 


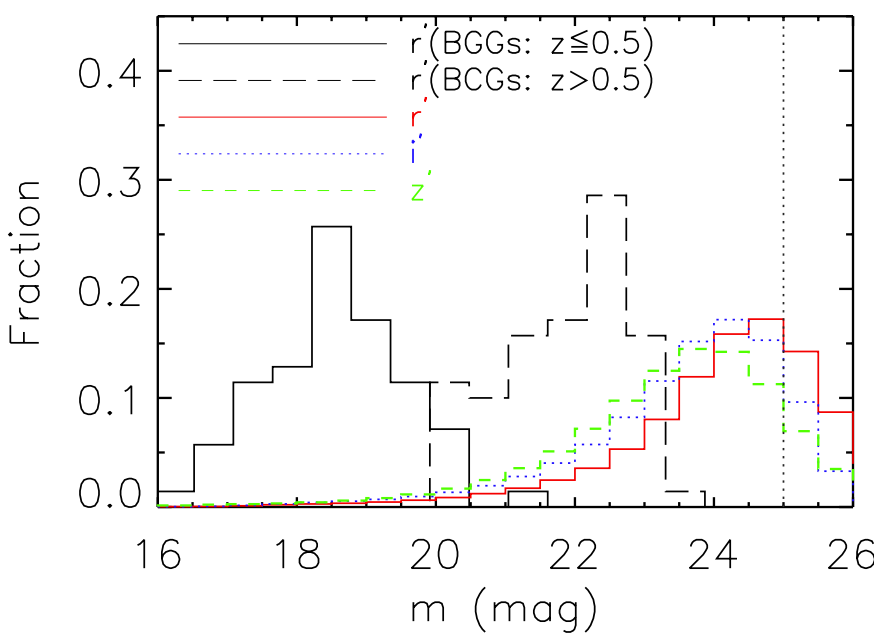

Fig. 3. Magnitude distribution of the CFHTLS-W1 photometric data in $r^{\prime}-, i^{\prime}$ - and $z^{\prime}$ band. The solid and dashed black histograms indicate the $r^{\prime}$-band magnitude distribution of BGGs and BCGs in our catalog in the redshift ranges, $z \leqslant 0.5$ and $z>0.5$, respectively. The vertical dotted black line represents the magnitude completeness of the CFHTLS data in $r^{\prime}$-band.

account for this effect in the plots, we assigned all $\Delta M_{1,2}>2$ systems to a single bin (see Fig. 12).

In this paper, we use the photometric redshift catalogs of the CFHTLS survey by Brimioulle et al. (2008, 2013). The XMM-CFHTLS W1 field, has a good spectroscopic coverage of $\sim 0.64$ degrees $^{2}$ with the VIMOS-VLT Deep Survey (VVDS; Le Fèvre et al. 2004, 2005) and the targeted cluster follow-up of Adami et al. (2011).

\section{Semi-analytic galaxy catalogs}

In order to decipher the observational trends it is instructive to compare them to predictions of theory. To this end we have embarked on the semi-analytic galaxy catalogs of B06, DLB07, and G11. All three models implemented on the dark matter halo merging trees obtained from the Millennium simulation which was described in detail in Springel et al. (2005).

The B06 model improves Durham SAMs (GALFORM; e.g., Cole et al. 2000; Benson et al. 2003) notably the black hole growth, AGN feedback (Kauffmann \& Haehnelt 2000) and disk instability (Cole et al. 2000; Mo et al. 1998). The DLB07 model is a developed SAM of those presented in Springel et al. (2005), De Lucia et al. (2006) and Croton et al. (2006) models. G11 is an updated version of these models.

To estimate the photometric properties of galaxies, DLB07 and G11 apply the stellar population synthesis model of Bruzual \& Charlot (2003) and the initial mass function (IMF) of Chabrier (2003). B06 uses a Kennicutt (1983) IMF with no correction for brown dwarf stars and outputs magnitudes in the Vega system, which we convert to the $\mathrm{AB}$ system to match two other models.

G11 refines the definition of a satellite galaxy in halos and treats a galaxy as a satellite, applying the tidal and ram-pressure stripping, only at times when it is located within $R_{\mathrm{vir}}$. In two other models a galaxy acts as a satellite when it is ascribed to a larger friends-of-friends halo and its hot gas atmosphere is immediately stripped, leading a reddening in color and a rapid decline in the star formation. Thus, modifications of G11 lead galaxies to retain more gas for star formation.

The merger trees (Harker et al. 2006) used in the B06 model differ from that implemented in DLB07 and G11
(Springel et al. 2005). All three models include star-bursts triggered by major merger, associated with the mass ratio of the merging progenitors in excess of 0.3 . When a major merger occurs, all the stellar objects in progenitors are transferred into the bulge of the remnant galaxy. In G11 and DLB07 a fraction of cold gas turns into the bulge stars (Somerville et al. 2001), while in B06 all the cold gas is converted into stars. Among these three models only G11 considers the satellite-satellite mergers, which can reduce the number of satellite galaxies in massive halos (e.g., Kim et al. 2009).

Another relevant physical process for galaxy evolution which has only been taken into account in G11 model is a tidal disruption of the stellar component from merging satellites (e.g., Font et al. 2008; Henriques \& Thomas 2010).

Following White \& Frenk (1991), all three SAMs apply a model for the cooling from hot gas halo. They all define a cooling radius to distinguish between the rapid cooling and static hot halo regimes and compare it with the virial radius (DLB07 and G11) or the free-fall radius (B06). B06 assumes that cooling flows in halos with virial velocities below $50 \mathrm{~km} \mathrm{~s}^{-1}$ are suppressed at $z \leq 6$ (see B06 and G11). In addition, all the models include the AGN feedback based on the model introduced by Kauffmann \& Haehnelt (2000) which suppresses cooling flows in massive halos. The feedback used in B06 is similar to the radio mode feedback considered in Croton et al. (2006), but the details of implementation are different. In B06 AGN feedback is more effective at low- $z$ during the quasi-hydrostatic phase. G11 and DLB07 apply both the radio mode and quasar mode following Croton et al. (2006). In G11 the radio mode feedback and gas cooling are also applied on massive satellite galaxies.

Finally, all three SAMs implement SNe feedback from massive stars, which can heat gas and eject it from the cold disc into the hot halo. G11 uses a model of the SNe feedback which depends on the galaxy circular velocity $\left(V_{\max }\right)$ and gives stronger feedback at low- $V_{\max }$ and dwarf galaxies.

In summary, treatments of both minor and major mergers as well as dry and wet mergers, cooling flows, AGN and stellar feedback can affect galaxy evolution and its properties (e.g., stellar mass, luminosity and stellar age). In this paper, we perform a statistical study of the redshift evolution of the magnitude gap out to $z=1.1$ to compare the galaxy properties in massive halos predicted by different SAMs and examine consistency with observations.

\section{Group identification}

\subsection{Red-sequence method}

The full details of our red sequence finder are presented in Mirkazemi et al. (2014), but here we provide an outline of the method. We measure an excess of red sequence galaxies over the background within the $0.5 \mathrm{Mpc}$ radius from the X-ray center. The background is computed using 200 random areas in the CFHTLS survey and repeating the selection of galaxies used in the red sequence. We define the red sequence significance as a ratio of the excess number density of red sequence galaxies to a dispersion in the number density of background galaxies. The redshift at which the red sequence significance reaches its maximum value is taken as the redshift of a cluster. Our red sequence filter is defined as sliding (as a function of redshift) upper and lower limits for galaxy colors and a selection of bright, $L \geq 0.4 L_{*}$, galaxies. Using Maraston et al. (2009) stellar population models for the red galaxies we derived the characteristic luminosity $L^{*}$ for the MegaCam $g^{\prime}, r^{\prime}, i^{\prime}$ and $z^{\prime}$ filters at each 


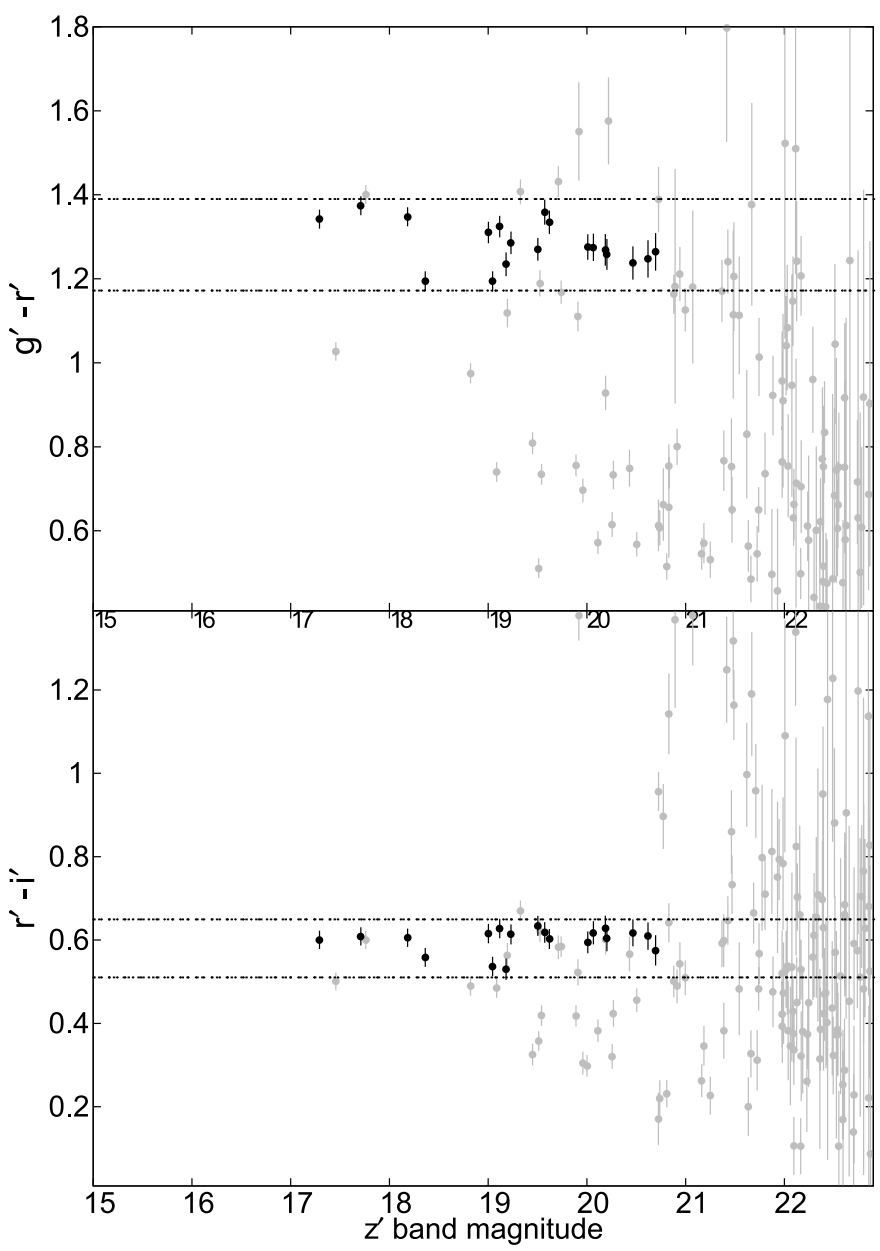

Fig. 4. Example of using two-color magnitude diagrams to identify the red sequence of a galaxy cluster at $z=0.28$ (cluster 100320). Top and bottom panels show $g^{\prime}-r^{\prime}$ and $r^{\prime}-i^{\prime}$ versus $z^{\prime}$-band magnitude, respectively. Gray points are all galaxies within the radius of $0.5 \mathrm{Mpc}$ from the X-ray source center. Black dots are red galaxy group members selected according to the method described in Sect. 4.1. The upper and the lower ranges of colors are shown with the horizontal dashed lines.

redshift. A model for the colors of red sequence galaxies is derived by a sample of red galaxies with spectroscopic data from the SDSS III (Aihara et al. 2011) and Hectospec (Mirkazemi et al. 2014). This sample of red galaxies gives a model for colors of red galaxies up to a redshift of 0.75 . Beyond this redshift, Maraston et al. (2009) stellar population model was used. The upper and lower ranges on colors of red galaxies were defined as $\pm 2 \Delta_{\mathrm{rs}}$ around the color model at each redshift. The dispersion $\Delta_{\mathrm{rs}}$ has two terms: an intrinsic color dispersion and the measurement uncertainty. The first term is modeled by varying the metallicity in the PEGASE. 2 stellar population models (Fioc \& Rocca-Volmerange 1999). To compute the second term, we use the error in measuring the magnitudes in the CFHTLS data, as detailed in Bielby et al. (2010).

We chose the following combination of filters as a function of redshift for the red sequence algorithm:

$0.05 \leq z \leq 0.66: g^{\prime}, r^{\prime}, i^{\prime}$

$0.66<z \leq 1.10: r^{\prime}, i^{\prime}, z^{\prime}$.

Figure 4 shows the $g^{\prime}-r^{\prime}$ and $r^{\prime}-i^{\prime}$ colors versus $z^{\prime}$ band magnitude for a cluster at a redshift of 0.28 .

For computing the absolute magnitude of red sequence galaxies, we applied a $\chi^{2}$ template-fitting method using the Le Phare package (Arnouts et al. 2002) and templates of

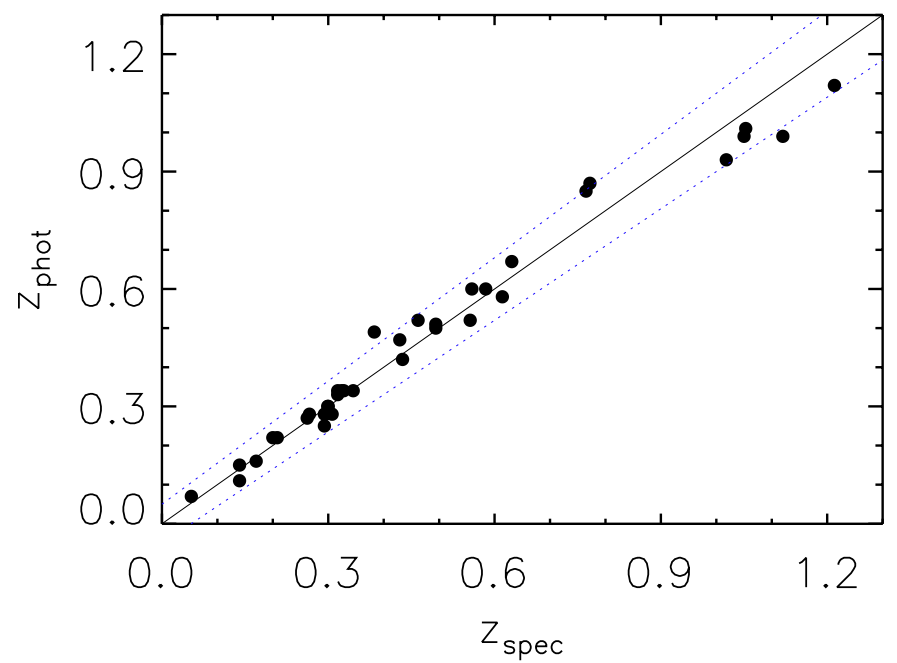

Fig. 5. Photometric redshift versus spectroscopic redshift for our sample of galaxy groups. Black line gives the 1:1 relation and the dotted blue lines show the adopted $\pm 0.05(1+z)$ uncertainty of our red sequence redshift.

Ilbert et al. (2006). We applied the Calzetti et al. (2000) extinction law to the star-forming, irregular and star burst templates. Absolute magnitudes of member galaxies were computed assuming the redshift of their host galaxy group. Similar to Ilbert et al. (2009) and Salvato et al. (2009), we applied the AUTOADAPT mode of the Le Phare code on a sample of galaxies with spectroscopic redshifts from the VVDS survey to reduce the systematic offset between photometric and spectroscopic redshifts by adjusting photometric zero points. Further details of the procedure can be found in Ilbert et al. (2006).

For galaxy groups, located within the VVDS survey, we compute the mean spectroscopic redshift of red sequence galaxies and identify as member the galaxies located within $R_{200}$ from the center of the group and obeying

$\left|z_{\text {member }}-z_{\text {group }}\right|<3 \frac{\sigma_{x}(v)}{c}$.

We estimate the galaxy velocity dispersion $\sigma_{x}(v)$ using the virial mass of groups (estimated from the X-ray luminosity), as introduced in Erfanianfar et al. (2013). In Table 1 we present the values of $R_{200}$ and $\sigma_{x}(v)$ for each galaxy group.

We were able to assign a spectroscopic redshift to 15 galaxy groups using the spectroscopic data of the VVDS survey. For further 30 groups and clusters in common with Adami et al. (2011) catalog, spectroscopic redshifts are adopted from that study. For the sample of spectroscopically identified systems, in Fig. 5 we compare the red sequence redshift to the spectroscopic one. The standard deviation between the red sequence and spectroscopic redshifts is 0.04 . Black line gives the 1:1 relation and blue dotted lines correspond to the adopted uncertainty in our red sequence redshift determination of $\pm 0.05(1+z)$.

\subsection{Visual inspection and identification of group candidates}

For identification of galaxy groups, assignment of a photometric redshift and determination of the contamination from several optical groups, we used the red-sequence technique as described in Sect. 4.1.

We visually inspected optical counterparts of each X-ray source using the photometric redshift catalog of the CFHTLS (Brimioulle et al. 2008, 2013) and the CFHTLS RGB image 

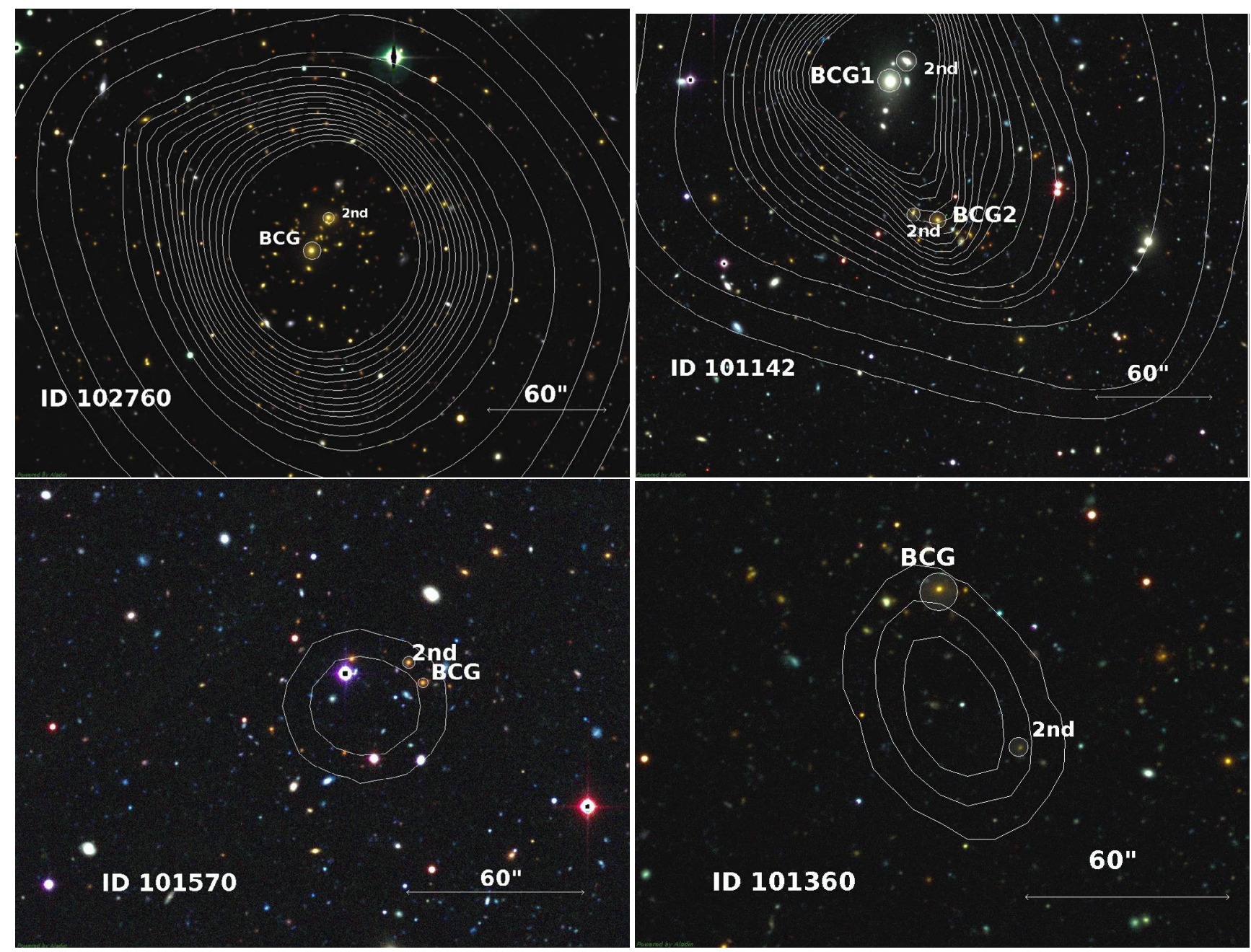

ID 101142
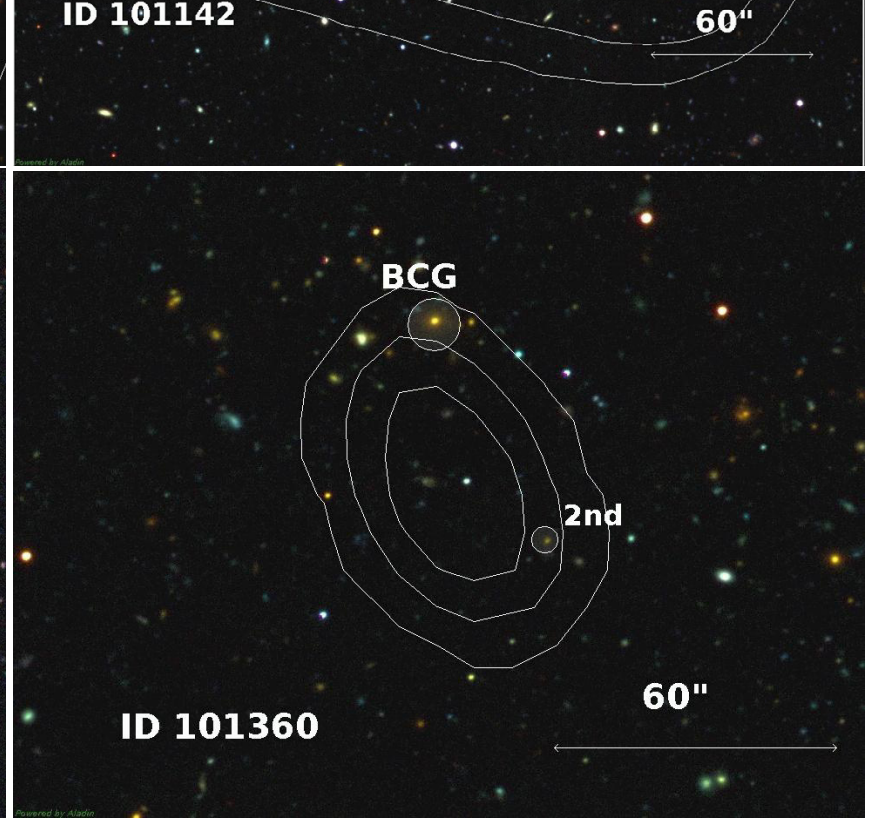

Fig. 6. Contours of the extended X-ray emission overlaid on the CFHTLS RGB image in $i^{\prime}, r^{\prime}, g^{\prime}$ filters. Top left panel shows a spectroscopically confirmed cluster at $z=0.47$ within the center of X-ray emission and having a single optical counterpart, with a high significance of X-ray flux estimate (flag $=1$ and flag $=3$ if without the spectroscopic confirmation). Top right panel, assigned with a flag $=2$, indicates a presence of two overlapping X-ray sources at $z=0.52$ and $z=0.15$. The bottom left image shows the multiple optical counterparts within the X-ray emission with no possibility of separating out their contributions (flag $=4)$. The bottom right panel shows a galaxy group at $z=0.60$ located at the edge of X-ray emission (flag =5). The scale bar is presented in each image.

in $i^{\prime}, r^{\prime}$ and $\mathrm{g}^{\prime}$ filters. The visual inspection helps us to assign a redshift to each $\mathrm{X}$-ray source, and to evaluate the correspondence between the galaxy distribution and the X-ray emission. For some sources where we found more than one counterpart and where the shape of X-ray emission allows us to separate the contribution from several counterparts, we specify a new ID in our X-ray source catalog.

We define a flag for each extended X-ray source based on the visual inspection and the position of the over-density of galaxies inside each X-ray source. We use flag values 1 and 3 to mark the best identifications. This requires having both a unique X-ray source with a well-defined center, and a unique optical counterpart with the spectroscopic confirmation for flag $=1$ or without one - for flag $=3$. A flag $=2$ is assigned when a single X-ray source has been split into several sources (e.g., top right panel in Fig. 6). A flag $=4$ indicates a presence of multiple optical counterparts, whose contribution to the observed X-ray emission is not possible to separate or rule out (e.g., bottom left panel in Fig. 6).

In cases where the X-ray emission covers a part of the group area and a concentration of galaxies is located at the edge of $\mathrm{X}$-ray emission, we assign a flag $=5$ (e.g., bottom right panel in
Fig. 6). Finally, systems with a potentially wrong assignment of optical counterpart are also flagged as 5.

\subsection{Catalog of identified groups}

We present a catalog of 129 X-ray selected galaxy groups in Table 1. Columns 1 and 2 indicate the internal X-ray source ID and our defined group ID. Columns 3 and 4 are the RA (J2000) and Dec (J2000) of the X-ray center. Column 5 shows the group photometric redshift estimated according to the method outlined in Sect. 4.1.

Column 6 is the total group mass, $M_{200 c}$, in $M_{\odot}$ and a corresponding statistical error, obtained using the $L_{\mathrm{x}}-M$ relation of Leauthaud et al. (2010). The systematic uncertainty on inferring $M_{200 c}$ using $L_{\mathrm{x}}$ is $20 \%$ (Allevato et al. 2012). Column 7 lists $L_{\mathrm{x}, 0.1-2.4}$ in the rest-frame $0.1-2.4 \mathrm{keV}$ energy range. Column 8 presents the galaxy velocity dispersion in $\mathrm{km} \mathrm{s}^{-1}$, estimated from $L_{X}$ using the scaling relations (Erfanianfar et al. 2013). Column 9 shows group's $R_{200 c}$ in degrees. Column 10 lists the mean group temperature in $\mathrm{keV}$ estimated using $L-T$ relation as in Finoguenov et al. (2007). 
G. Gozaliasl et al.: Evolution of the luminosity gap in X-ray galaxy groups

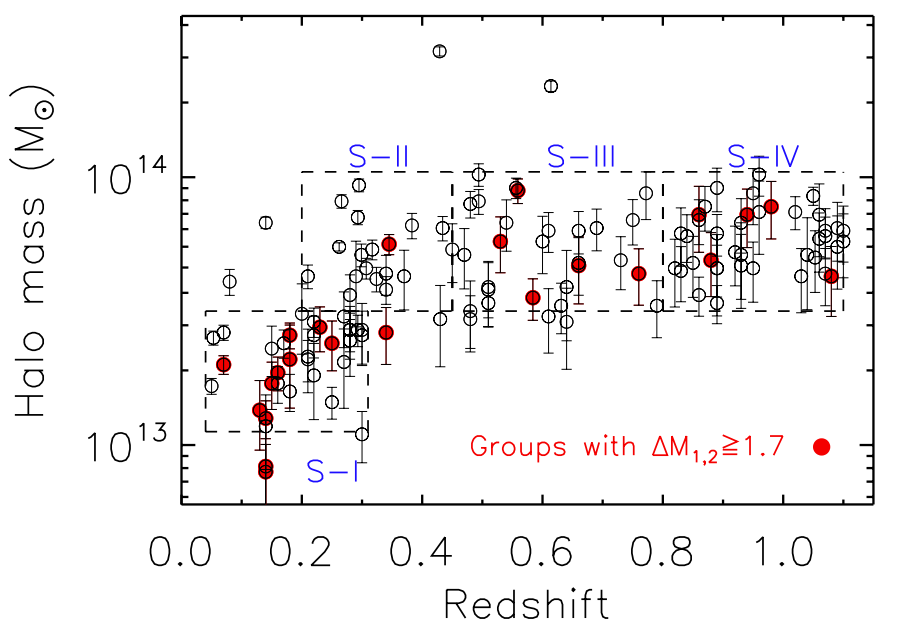

Fig. 7. Halo mass versus redshift for the detected X-ray groups. Groups with $\Delta M_{1,2} \geq 1.7$ have been marked with red filled circles. The dashed boxes show the four subsamples, defined in Sect. 4.3.

The group X-ray flux in the observed $0.5-2 \mathrm{keV}$ band (in units of $10^{-14} \mathrm{ergs} \mathrm{cm}^{-2} \mathrm{~s}^{-1}$ ) and a corresponding $1 \sigma$ error is listed in Col. 11. Column 12 provides the significance of the flux estimate. Column 13 reports the visual flag as defined in Sect. 4.2. Column 14 reports the spectroscopic redshift, available for 45 groups. We mark the spectroscopic redshift of groups adopted from with Adami et al. (2011) with ' $*$ '.

To test the evolution of properties of galaxy groups in observations and SAMs, we define four subsamples using the following redshift and halo mass ranges:

(S-I) $0.04<z<0.31$ and $13.00<\log \left(\frac{M_{200}}{M_{\odot}}\right) \leq 13.45$
(S-II) $0.20<z \leq 0.45$ and $13.45<\log \left(\frac{M_{200}}{M_{\odot}}\right) \leq 14.02$
(S-III) $0.45<z \leq 0.80$ and $13.45<\log \left(\frac{M_{200}}{M_{\odot}}\right) \leq 14.02$
(S-IV) $0.80<z \leq 1.10$ and $13.45<\log \left(\frac{M_{200}}{M_{\odot}}\right) \leq 14.02$.

S-I includes 30 galaxy groups, and other three subsamples, S-II, S-III and S-IV, contain 23, 29 and 41 massive groups, respectively. In Fig. 7, we illustrate subsamples with dashed boxes.

\subsection{Abundance of galaxy groups}

In order to study the abundance of galaxy groups in our survey, we compute the survey volume using the sensitivity toward the group detection as a function of coordinate in four selected redshift intervals. The components of the calculation consist of the countrate limits as a function of coordinate and a predicted countrate from a galaxy group given its redshift and mass in the $16^{\prime \prime}-128^{\prime \prime}$ aperture defined by the detection scales. The X-ray luminosity of the group is computed for each mass and redshift using the Leauthaud et al. (2010) scaling relation, and the surface brightness profile of the group is computed using the parametrization of Finoguenov et al. (2007). The countrate limits correspond to a four sigma deviation in the background RMS predicted on the scale of the detection, which include the local residual background estimate by the wavelets and the systematic errors on AGN removal. The analysis of the noise of the image on the detection scales has been presented in Connelly et al. (2012) and its negative side is well described by the Gaussian. Figure 8 shows the computed survey volume of the X-ray galaxy groups as a function of the halo mass, $M_{200}$, for different subsamples.

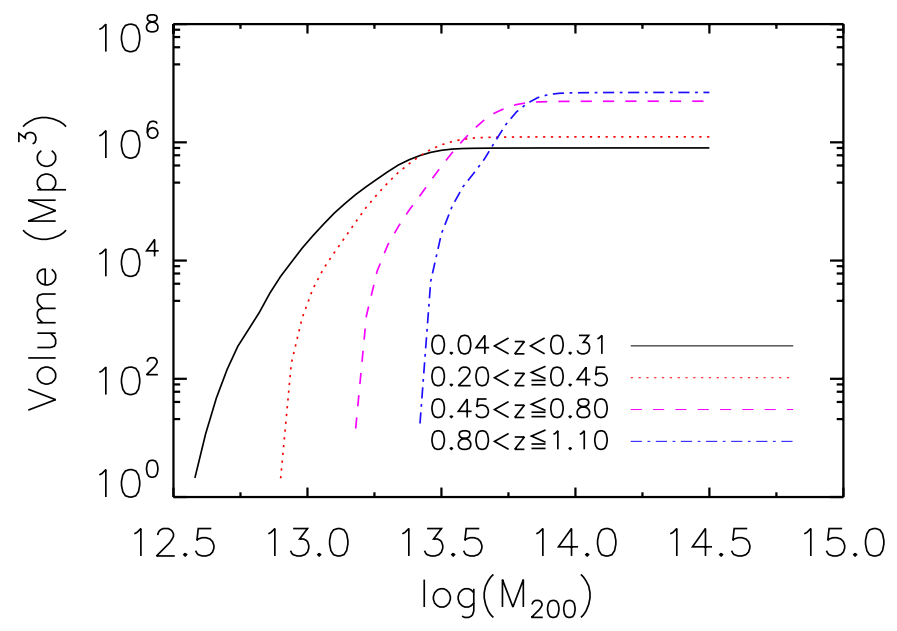

Fig. 8. Survey volume as a function of halo mass. Solid black, dotted red, dashed magenta and dash-dotted blue curves present trends for the four redshift intervals, S-I to S-IV, which are described in Sect. 4.3.

In Fig. 9 we compare the number density of galaxy groups as a function of halo mass (solid black histogram with error bar) with that from the SAMs of G11 (red dotted histogram), B06 (dash-dotted blue histogram) and DLB07 (dashed green histogram). Number of observed galaxy groups in each halo mass bin is normalized to the corresponding volume as shown in Fig. 8. For the normalization of the number of galaxy groups in the models we use the constant volume of the Millennium simulation, a box of size $500 \mathrm{~h}^{-1} \mathrm{Mpc}$ on a side. Halo abundance in models is consistent with the observed trend for S-I (left top panel). For S-II (top right panel ) and S-III (bottom left panel), this consistency becomes worse at high masses, but the agreement reappears at higher redshift traced by the subsample S-IV (bottom right panel).

A detailed assessment of the cosmological importance of the observed halo abundances is outside the scopes of this paper and would need to include the effect of the sample variance at high halo masses. The main conclusion we draw is that for further comparison we should rather use fractions determined separately for the group catalogs in observations and simulations.

\section{Membership contamination and magnitude gap calculation}

\subsection{Contamination of group membership}

The purity of the galaxy selection using the red-sequence method is important for calculating the magnitude gap of galaxy groups. In the absence of spectroscopic group membership, the calculation using red sequence galaxies may be contaminated by dusty star forming galaxies (DSFGs).

We examine the impact of contamination of group members by DSFGs using the X-ray galaxy group catalog in the redshift range $0.066<z<1.544$ presented in Erfanianfar et al. (2013) and the data of galaxies in the AEGIS Herschel survey located within a CFHTLS field covering the redshift range $0.05<z<1.10$. We define as DSFGs those galaxies which have star formation rate above $\approx 100 M_{\odot} \mathrm{yr}^{-1}$ as inferred from FIR observations by Herschel space telescope (Erfanianfar et al. 2014).

For each group in our CFHTLS catalog we test whether any of DSFGs would be selected as a member. We apply a luminosity cut of $L>0.4 L^{*}$, and use two red-sequence finders: a single $r^{\prime}-i^{\prime}$ color, and our two-color selection (Sect. 4.1). The 

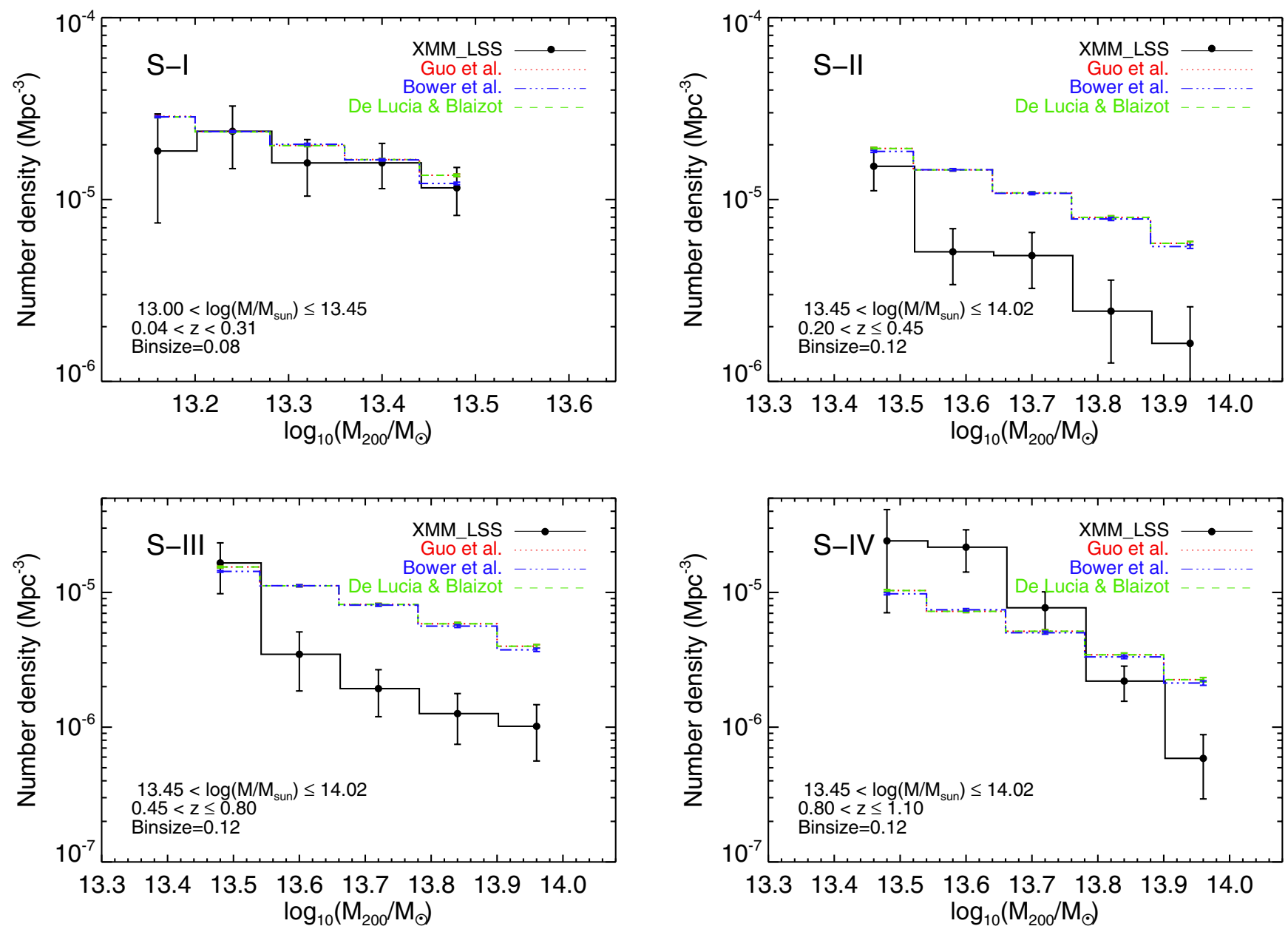

Fig. 9. Observed number density of galaxy groups as a function of halo mass (black histogram with error bars) compared to the halo catalogs of B06, DLB07 and G11 for S-I (top left panel), S-II (top right panel), S-III (bottom left panel) and S-IV (bottom right panel). Number density of halos in B06, G11 and DLB07 have been plotted with dash-dotted blue, dotted red and dashed green histograms and are identical. Halo abundance in simulations is consistent with the observed number density of galaxy groups in S-I, but this consistency is reduced at high masses for S-II and S-III. Subsamples are defined in the legend of each plot.

contamination for each group is estimated by multiplying the number of DSFGs selected by the red sequence method to the ratio of the area of group which was used to define the magnitude gap $\left(0.5 R_{200}\right)$ and the area of the Herschel data coverage of the AEGIS field, $\sim 0.35$ degrees $^{2}$. Figure 10 shows the expected contamination for each group versus its redshift for the single color and the two color selection of the group members. Two color selection completely removes the contamination at $z<0.6$. The expected contamination by DSFGs at $0.6<z<1.1$ is low, $\$ 3 \%$, to significantly affect the magnitude gap measurement.

To examine the effect of the contamination by DSFGs on the magnitude gap calculation, we use data of nine galaxy groups with spectroscopic membership from the AEGIS X-ray groups catalog within the redshift range of $0.65<z<1.1$ (Erfanianfar et al. 2013, 2014). The redshift range of these groups of galaxies covers the range where the contamination by DSFGs is the highest. Although, the redshifts of these groups do not span the full redshift range of our catalog, they can still give us a good estimate of contamination of the star forming foreground or background galaxies to the red-sequence. For these nine groups, we only find two galaxies as DSFGs which are group members, but they are not selected as a brightest cluster galaxy (BCG) or the second brightest satellite. Thus, in this test we also find that the contamination by DSFGs has no effect on the magnitude gap estimate.
In addition, one way to inspect the effect of the red sequence selection on the magnitude gap estimate is to compare values of the magnitude gap which are calculated using the photometric membership to that of the spectroscopic membership.

We use the X-ray group catalogs from the AEGIS field (Erfanianfar et al. 2013), the COSMOS field (Finoguenov et al. 2007; George et al. 2011), including all spectroscopic observations, available within the collaboration. We also use all spectroscopic data of groups in our catalog provided by the VVDS data and Adami et al. (2011).

We only consider the $\sim 1$ degree $^{2}$ area of the COSMOS survey, that is in common with CFHTLS survey, where a comparison to our red sequence selection using CFHT colors is possible. Altogether, we obtain a catalog of 84 galaxy groups (42 groups from the COSMOS catalog, 26 groups from our catalog and 16 galaxy groups from the AEGIS catalog), having the spectroscopic confirmation of the magnitude gap. These groups span a redshift range of $0.05<z<1.22$, which is similar to the redshift range of our XMM-LSS catalog.

For this sample of 84 groups (COSMOS+AEGIS+XMMLSS) we select the red sequence and spectroscopic members of groups according to the approach described in Sect. 4.1. We compute the magnitude gap between the first and the second brightest group galaxies using both spectroscopic and 


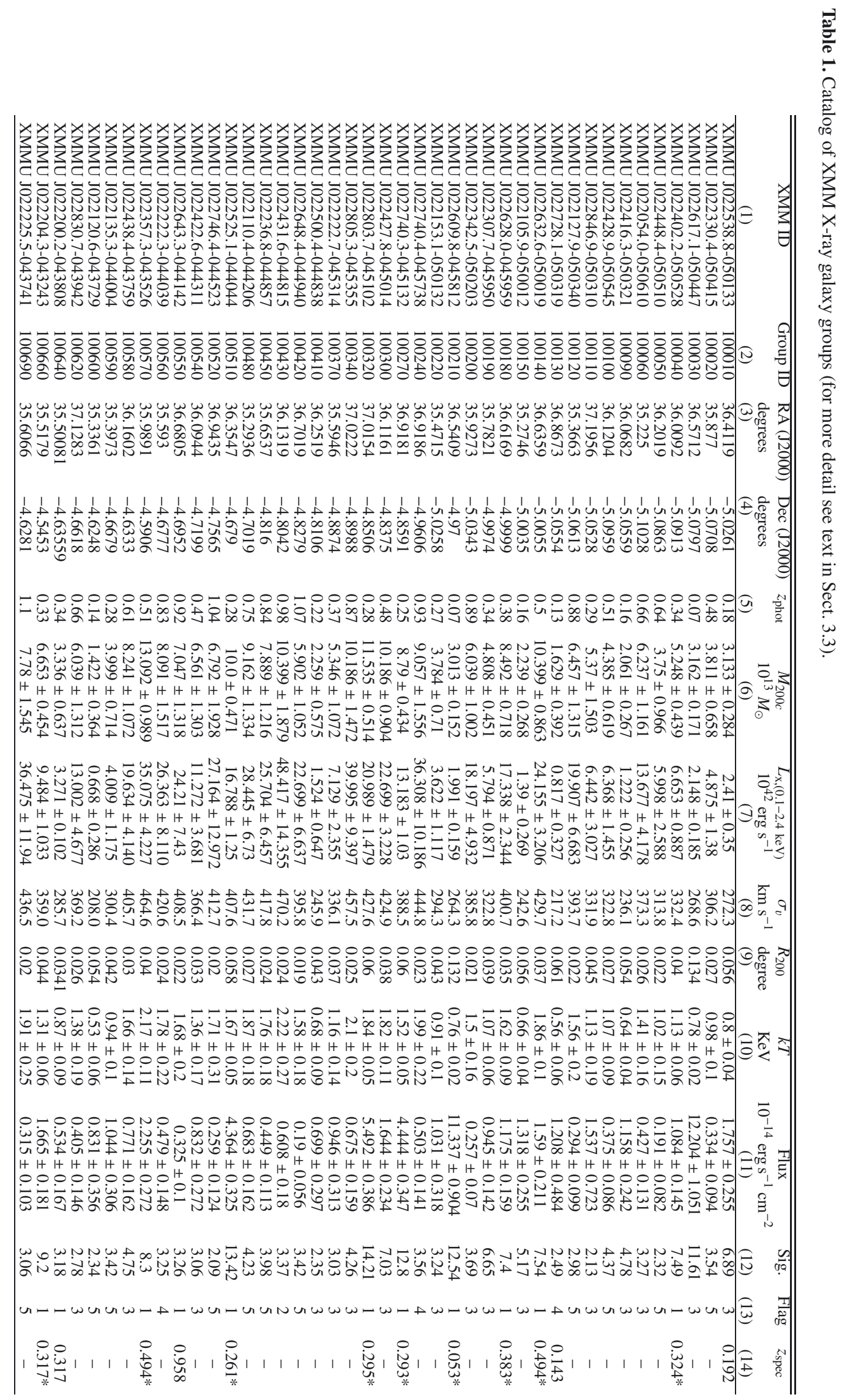




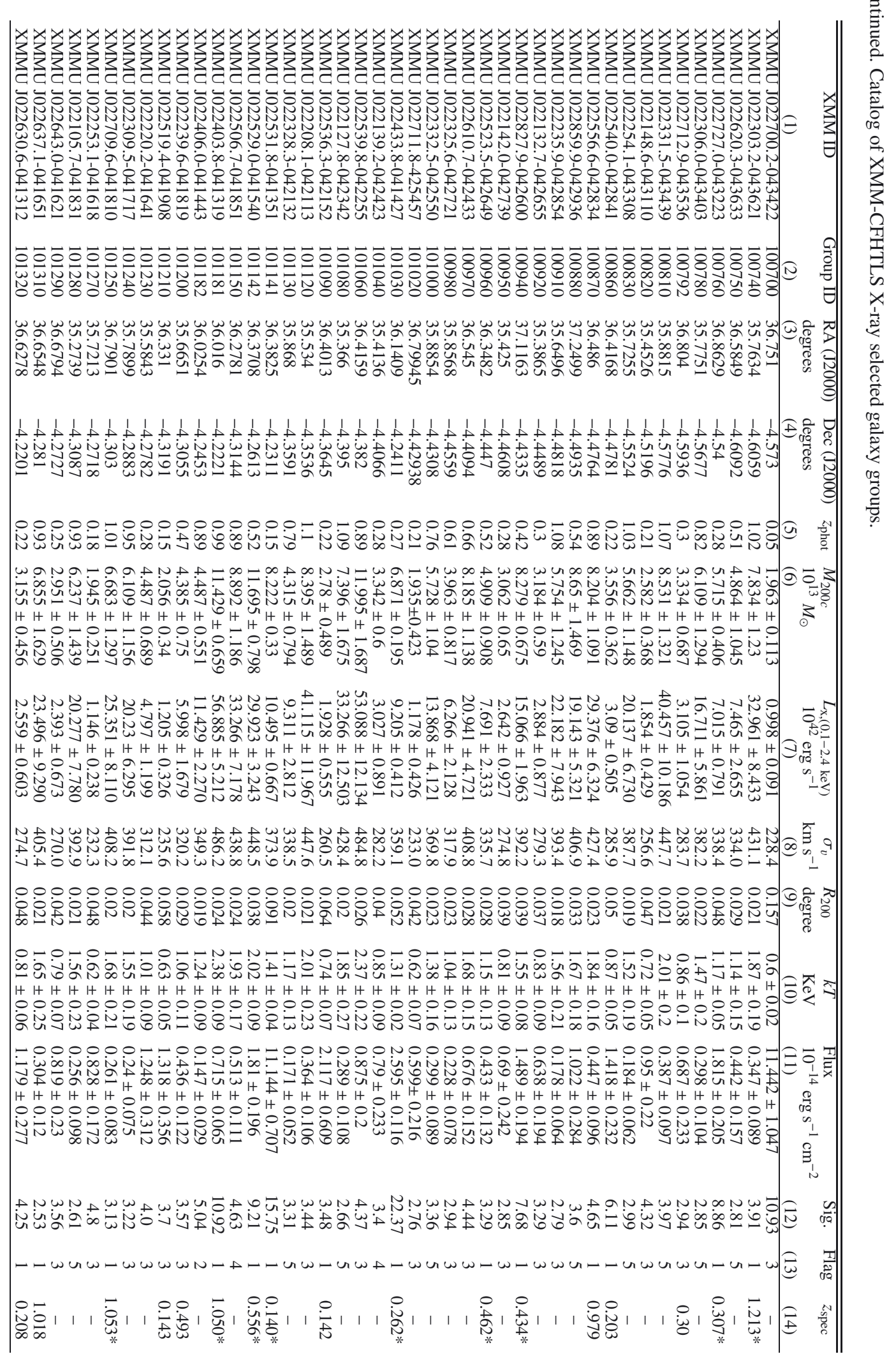




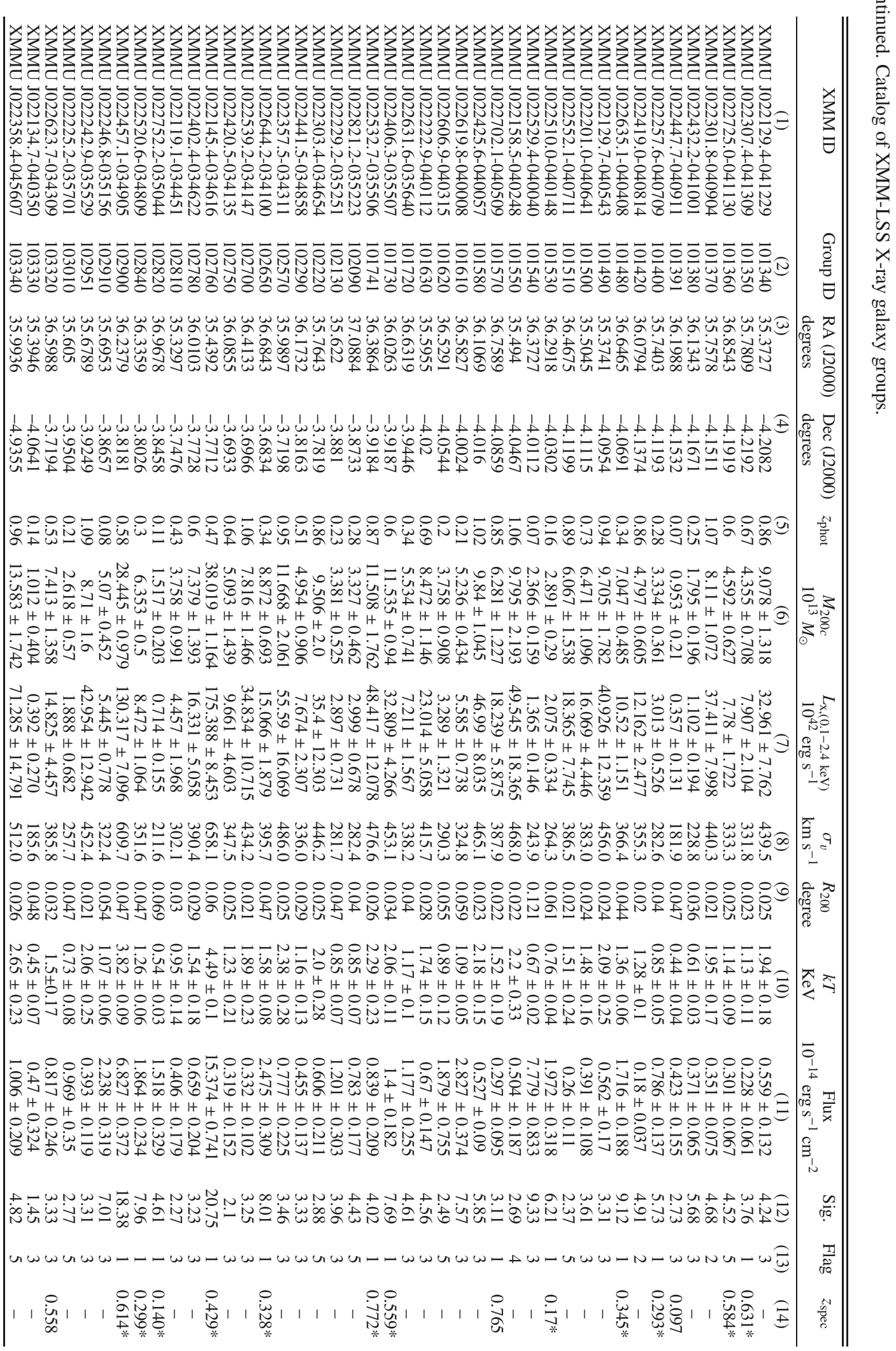




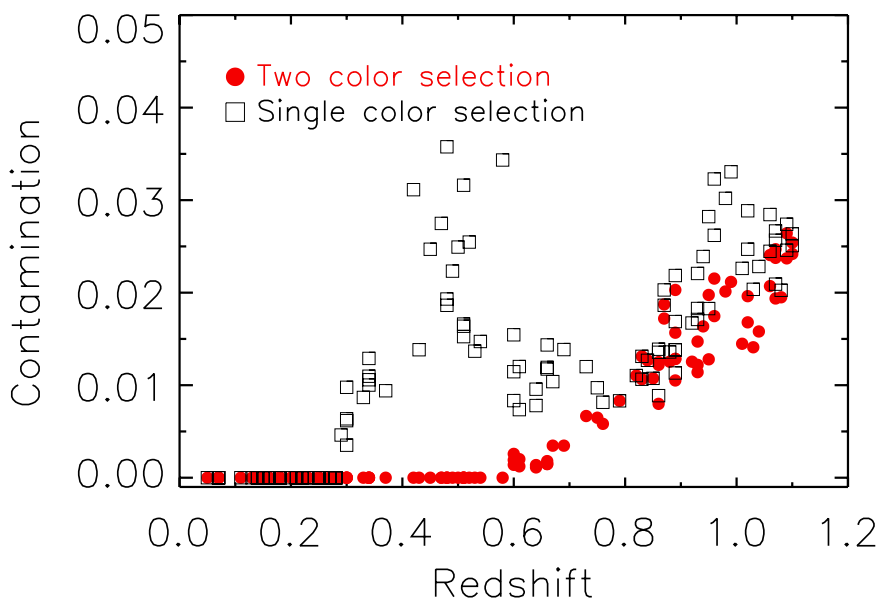

Fig. 10. Model prediction for red sequence contamination by dusty starburst galaxies versus redshift of group. The filled red circles and open black squares show the contamination for the two- and single-color selection of group members.

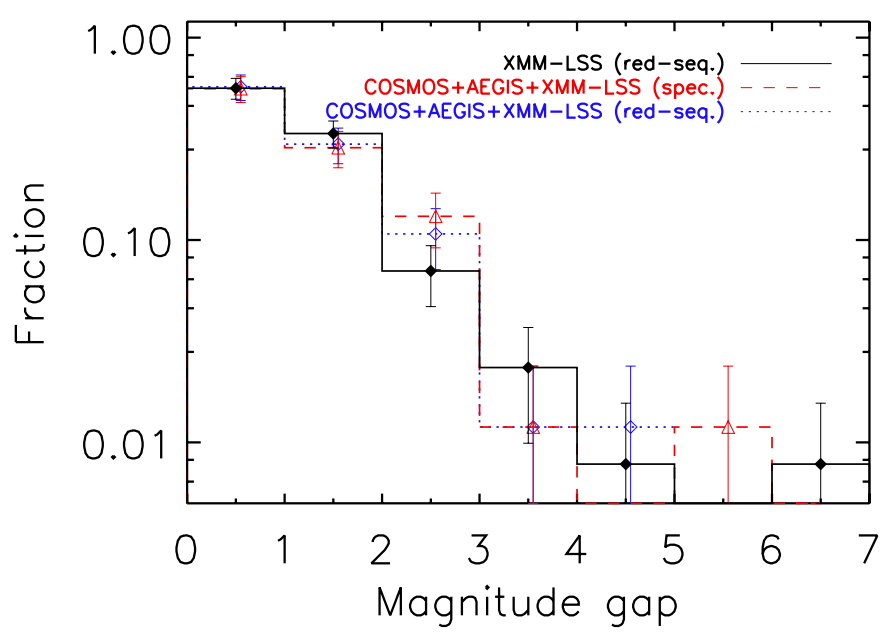

Fig. 11. Comparison of the magnitude gap distributions between our $\mathrm{X}$-ray group catalog (XMM-LSS) with the red sequence membership (solid black histogram) and $84 \mathrm{X}$-ray galaxy groups from the COSMOS+AEGIS+XMM-LSS with the spectroscopic membership (dashed red histogram) and the red sequence membership (dotted blue histogram). The redshift range is $0.05<z<1.22$. A good agreement is seen between the three distributions, implying that the results obtained with the red sequence selection are robust.

photometric group members, separately. The magnitude gaps of the spectroscopic sample range from 0.02 to 5.75 .

In Fig. 11 we compare the magnitude gap distribution of the COSMOS+AEGIS+XMM-LSS groups for two calculations of gap, one - using red sequence membership (dotted blue histogram) and the other - using the spectroscopic membership (dashed red histogram). It appears that the two distributions do not deviate considerably. In Fig. 11 we also compare these distributions to that of our XMM-LSS galaxy groups catalog with red sequence members (solid black histogram).

By comparing the spectroscopic and photometric membership assignment for the COSMOS+AEGIS+XMM-LSS groups, we find that redshifts of the second ranked red sequence galaxies in six groups deviates from the redshift of the hosting galaxy group. The magnitude gaps of these contaminated groups, which have been computed using red sequence galaxies range as follows: three galaxy groups with $\Delta M_{1,2} \geq 1.7$ (classified as fossils) and three galaxy groups with $\Delta M_{1,2} \leq 1.28$ (classified as non-fossils). Differences between the gap values in two calculations range from 0.05 to $1.2 \mathrm{mag}$. These deviations do not affect the classification by $\Delta M_{1,2}$ in 5 out of 6 cases. From finding one system to change the classification, we place a $95 \%$ confidence limit on the effect of $<6 \%$, based on the Poisson PDF. We added the uncertainty associated with this to each magnitude bin. Fractionally the effect is strongest for the bin containing the large magnitude gap systems.

\subsection{Determination of $\Delta M_{1,2}$ and definition of fossil groups}

In a number of studies different fossil group criteria for the magnitude gap and search radius have been used. For instance, La Barbera et al. (2012) defined as fossil groups those elliptical galaxies with no satellite brighter than a given magnitude gap of $\Delta M_{1,2} \geq 1.75$, within a search radius of $0.35 \mathrm{Mpc}$ and a maximum redshift difference of 0.001 . Voevodkin et al. (2010) classified an object as a fossil group if $\Delta M_{1,2} \geq 1.7$ for galaxies inside $0.7 r_{500}$. Smith et al. (2010) used a fixed projected physical radius of $R=640 \mathrm{kpc}\left(\sim 0.4 r_{200}\right)$ for a sample of massive clusters at $z \lessgtr 0.3$ to study the distribution of luminosity gap.

We define the spatial selection of galaxies for the $\Delta M_{1,2}$ calculation in such a way that it allows us to select a subsample of fossil groups candidates. For computing the magnitude gap of a galaxy group, we adopt the coordinates of the BGG as a center of group and measure the magnitude gap, $\Delta M_{1,2}$, using the rest frame absolute $r$-band magnitude of galaxies between the BGG and the second brightest galaxy within $0.5 R_{200}$, following Jones et al. (2003). The same definition of the magnitude gap has been applied to the SAM catalogs.

We define as a fossil group candidate, the X-ray group of galaxies that has a magnitude gap exceeding $\Delta M_{1,2} \approx 1.7 \mathrm{mag}$ within $0.5 R_{200}$. The low $L_{\mathrm{x}}$ cut has been historically done to avoid inclusion of X-ray emitting galaxies. However, by implying this cut one restricts the studies only to massive groups. Since we remove the galactic contribution to the X-ray emission by using only large spatial scales in the flux estimates, we do not have to limit the selection of fossil groups. Our lowest $L_{\mathrm{x}}$ group has a luminosity in the rest-frame $0.1-2.4 \mathrm{keV}$ band of $(3.6 \pm 1.3) \times 10^{41} \mathrm{ergs} \mathrm{s}^{-1}$.

\section{Results and discussion}

\subsection{Magnitude gap distribution}

We calculate the magnitude gap between the first and the second brightest galaxies in groups and clusters according to the method described in Sect. 5.2. For 26 groups in our catalog we calculate the magnitude gap using a spectroscopic membership assignment and for the remaining 102 groups the gaps are calculated using the red-sequence membership assignment.

In Fig. 12, we compare the distribution of the magnitude gap between X-ray groups and the predictions of SAMs, G11, DLB07 and B06. As discussed in Sect. 2.2, the completeness limit may affect the magnitude gap calculation of groups with $\Delta M_{1,2}>2$ at $z>0.5$. To account for this effect we define two different bin sizes, with 1 mag for systems with $\Delta M_{1,2}<2$, while assigning all $\Delta M_{1,2} \geq 2$ groups to a single bin (see Fig. 3 ). Such a binning scheme is introduced to remove the effect of completeness on the binned fractions.

For SAMs we use both the formal selection of the subsamples (solid histograms) and modeling of the X-ray selection. 
G. Gozaliasl et al.: Evolution of the luminosity gap in X-ray galaxy groups
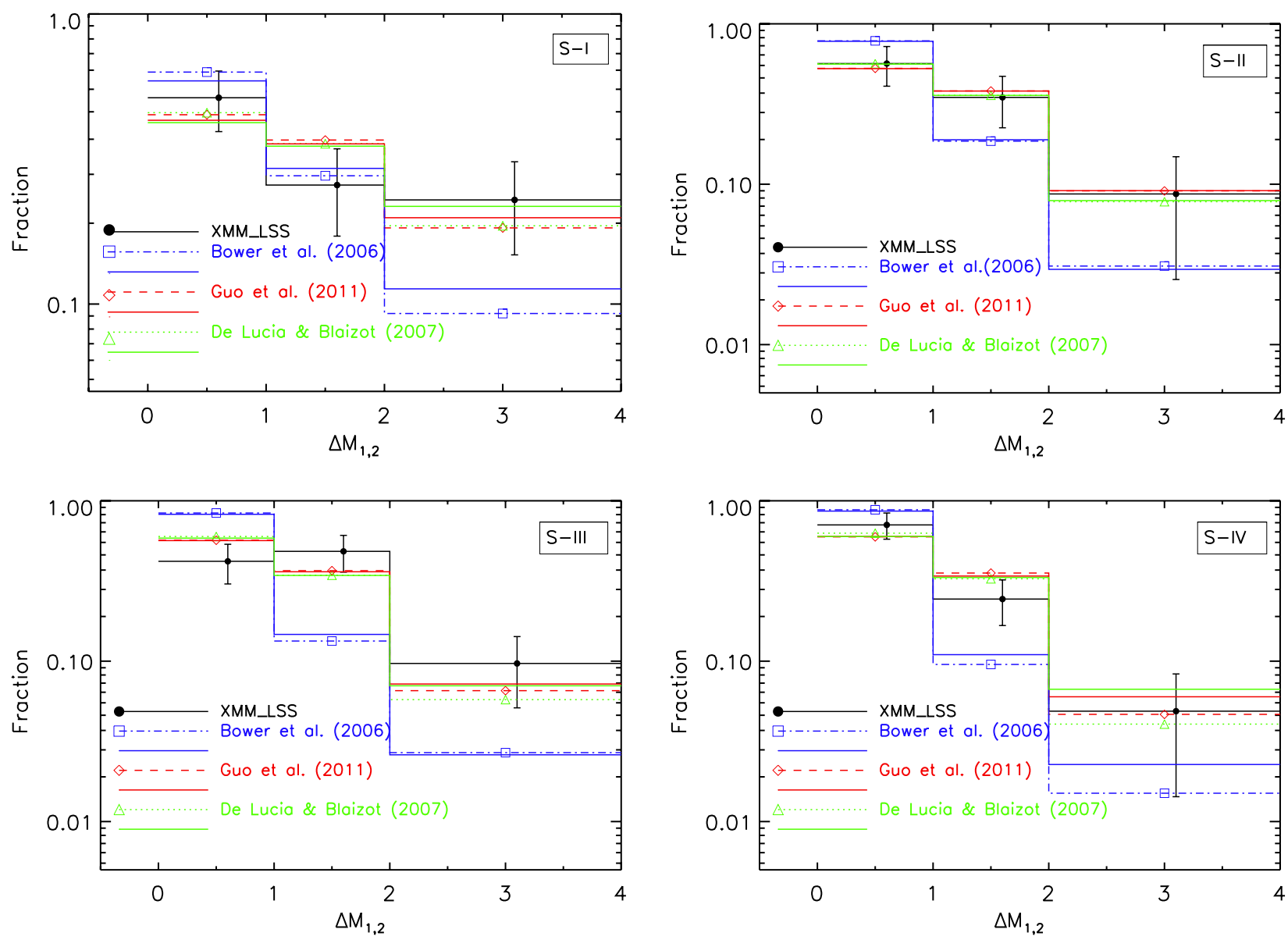

Fig. 12. Observed fraction of galaxy groups as a function of their magnitude gap (black points with error bars) compared to the predictions of semi-analytic galaxy formation models, B06 (solid and dash-dotted blue histograms), DLB07 (solid and dotted green histograms) and G11 (solid and dashed red histograms) for S-I (top left panel), S-II (top right panel), S-III (bottom left panel) and S-IV (bottom right panel). We find that the fraction of groups declines with magnitude gap at all redshifts. Magnitude gap bin sizes are equal to one magnitude for $\Delta M_{1,2}<2$. $\Delta M_{1,2} \geq 2$ systems are plotted as a 2 mag wide bin.

For the later, we introduce mass-dependent weights, $\frac{V_{\text {obs }}\left(M_{200}, z\right)}{V_{\text {sim }}}$. $V_{\text {obs }}$ is the survey volume for detecting groups as a function of $M_{200}$ and $z$, where $V_{\text {sim }}$ is equal to the $\left(500 \mathrm{Mpc} h^{-1}\right)^{3}$ volume of the Millennium simulation. We evaluate the total number of groups corresponding to each $\Delta M_{1,2}$ bin by summation of the weighted number of groups. We illustrate the so constructed model magnitude gap distributions with dashed red (G11), dashdotted blue (B06) and dotted green (DBL07) histograms in Fig. 12. It can be seen that the differences in the resulting magnitude gap distributions are small compared to our observational errors.

In agreement with the findings of Smith et al. (2010), the fraction of detected groups, $f\left(\Delta M_{1,2}\right)$, in all histograms of Fig. 12 declines with $\Delta M_{1,2}$. A linear relation provides a good fit to the data. We provide the fit parameters corresponding to each subsample in Table 2. The slope of the relation is shallower for (S-I) compared to other subsamples, indicating a larger contribution of fossil groups. The trends seen in the data for groups and clusters with $\Delta M_{1,2} \leqslant 3$ are well reproduced by both DLB07 and G11 models, while the B06 model predicts the slopes that are too steep. In addition, as previously noticed by Dariush et al. (2010), the B06 model over-predicts the fraction of clusters with $\Delta M_{1,2} \leqslant 1$ for S-II to S-IV.

In Fig. 13, we investigate a mass dependence of the fraction of groups characterized by a large magnitude gap $\left(\Delta M_{1,2} \geq 1\right)$.
Table 2. Parameters of the linear fit to a distribution of groups over the value of magnitude gap, $\Delta M_{1,2}$.

\begin{tabular}{lcc}
\hline \hline Subsample & Intercept & Slope \\
\hline S-I & $0.51 \pm 0.12$ & $-0.11 \pm 0.06$ \\
S-II & $0.70 \pm 0.01$ & $-0.22 \pm 0.01$ \\
S-III & $0.57 \pm 0.14$ & $-0.14 \pm 0.07$ \\
S-IV & $0.75 \pm 0.17$ & $-0.25 \pm 0.09$ \\
\hline
\end{tabular}

The relation reveals a mild dependence on mass, justifying our use of wide mass bins.

Figure 14 summarizes the trends presented in Fig. 13 and shows the redshift evolution of the mean fraction of large magnitude gap groups/clusters, $n(z)$. We find that $n(z)$ evolves slowly with redshift: $n(z)=0.47-0.12 \times z$, in agreement with all models. B06 underestimates fraction of clusters having a large magnitude gap.

Behavior of the luminosity gap in groups and clusters can depend on several effects, which we describe below. If a galaxy brighter than the second brightest galaxy falls into the cluster or group, the luminosity gap of the system changes to a lower value, as soon as the infalling galaxy passes into $\left(0.5 R_{200}\right)$, within which the $\Delta M_{1,2}$ is calculated. For the galaxy group members, the dynamical friction causes satellite galaxies to merge together or with the central galaxy. Mergers between satellites can reduce 

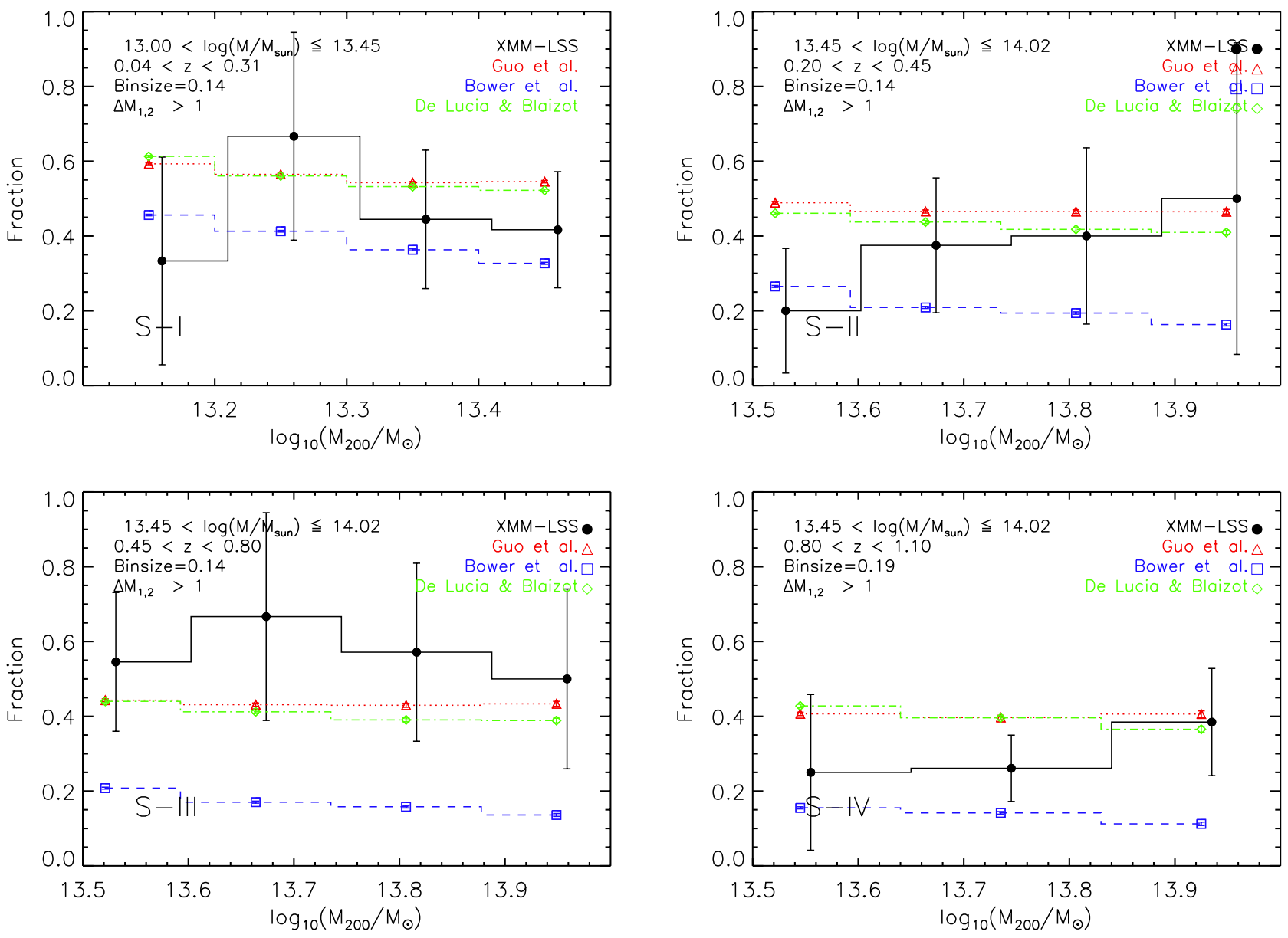

Fig. 13. Halo mass dependence of the fraction of groups with $\Delta M_{1,2}>1$. Black points with error bars show the data and dotted red, dashed blue and dash-dotted green histograms present model predictions of G11, B06 and DLB07, respectively for S-I (top left panel), S-II (top right panel), S-III (bottom left panel) and S-IV (bottom right panel). While the data are in agreement with the models, the low statistics of the data prevents us from choosing the best model.

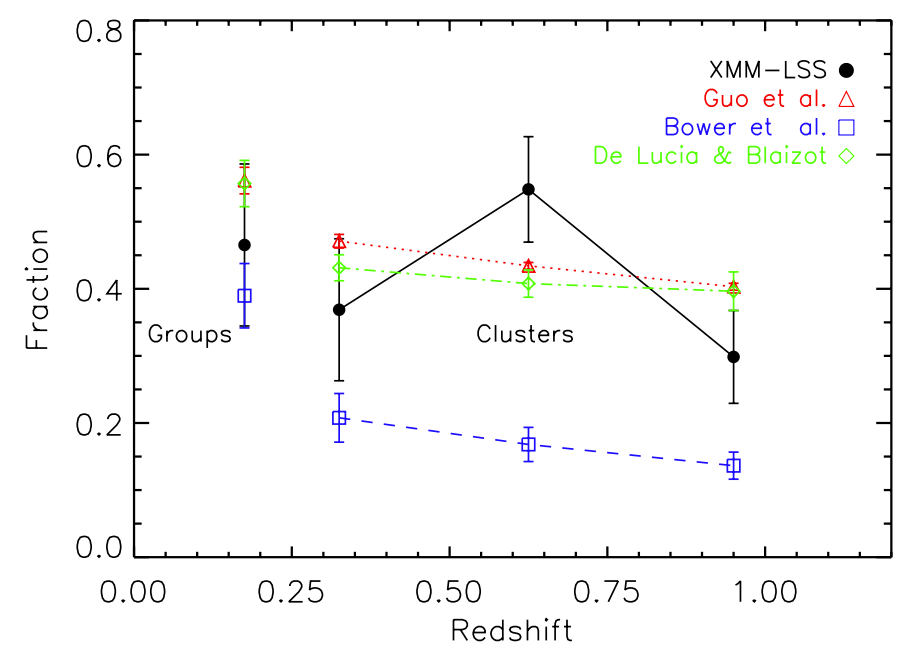

Fig. 14. Redshift evolution of the fraction of groups and clusters with $\Delta M_{1,2}>1$. The error bars correspond to the $95 \%$ confidence limits of the standard error of the mean.

the number of satellite galaxies in SAMs and in massive halos, especially reducing the number of the low-luminosity satellite galaxies; the product of merger is more luminous and massive. Finally, evolution of galaxy properties, most notably the stellar mass and age of the stellar population in both central and satellite galaxies also affects the magnitude gap. This in turn depends on the gas cooling rate, tidal stripping and disruption, $\mathrm{AGN}$ and $\mathrm{SNe}$ feedback.

The G11 model shows the best agreement with our observational results, possibly due to its advances in modeling the satellite disruption and gas striping due to the tidal and ram-pressure forces and the satellite-satellite merger. These processes are not taken into account in B06 and DLB07, but play an important role in the evolution of the galaxy luminosity in massive halos (Conroy et al. 2007a,b; Henriques \& Thomas 2010; Liu et al. 2010).

\subsection{Magnitude gap and BCG luminosity relation}

\subsection{1. $M_{r}-\Delta M_{1,2}$}

In Figs. 15 to 19 we plot the absolute magnitudes of the first $\left(M_{r, 1}\right)$ and the second $\left(M_{r, 2}\right)$ brightest galaxies against $\Delta M_{1,2}$. Previously, such butterfly diagrams have been used to quantify the comparison between observations and predictions of the SAMs below the redshift of $\sim 0.3$ (Smith et al. 2010; Tavasoli et al. 2011). In this paper, we extend these studies to $z<1.10$.

In Sect. 5.1 we examined whether contamination can affect the gap computations when the red-sequence method is applied for the selection of group membership versus the spectroscopic selection. In Fig. 15 we examine this effect on the butterfly 
G. Gozaliasl et al.: Evolution of the luminosity gap in X-ray galaxy groups
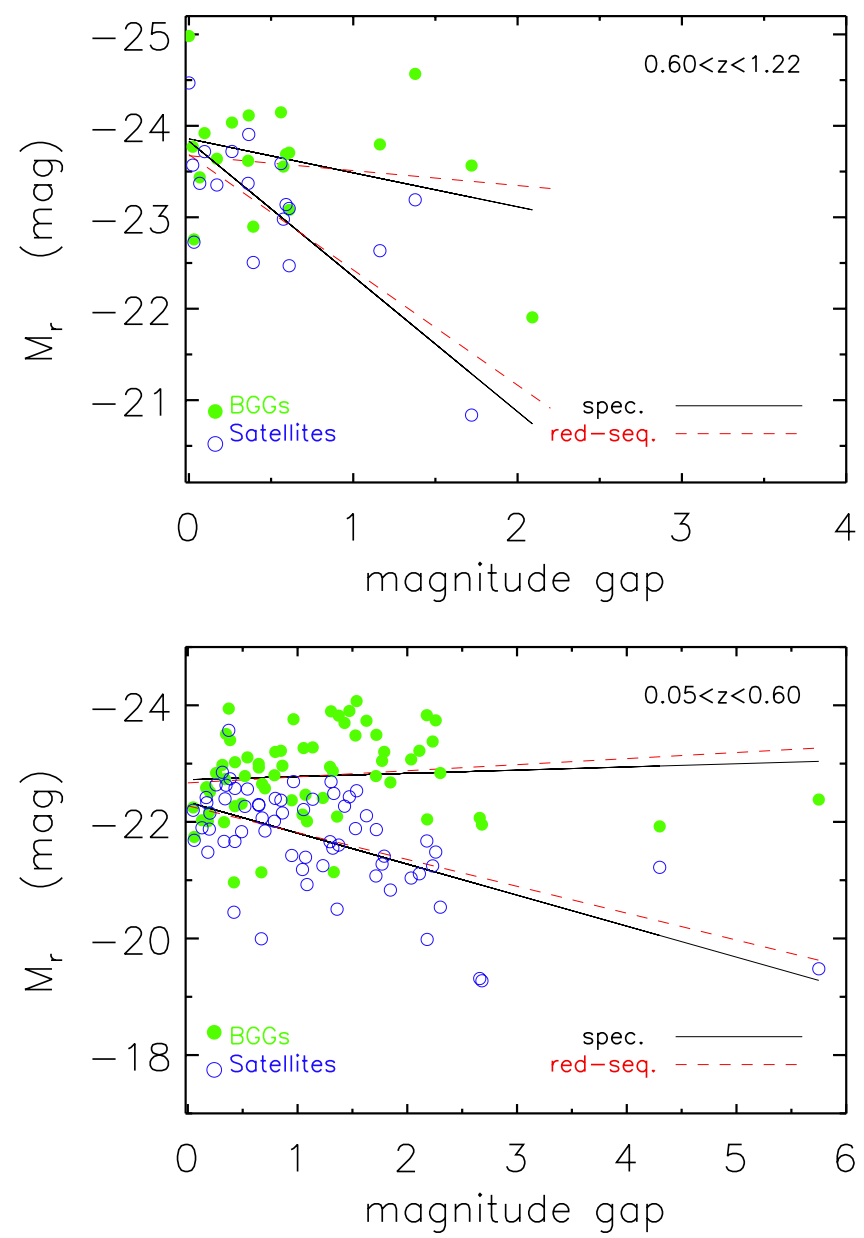

Fig. 15. Absolute $r$-band magnitudes of the first (filled green circles) and the second (open blue circles) brightest galaxies as a function of the magnitude gap for the COSMOS+AEGIS+XMM-LSS sample of groups, having spectroscopic members. The solid black and dashed red lines show linear fits for the spectroscopic and red sequence selections of group membership, respectively. The upper panel and lower panel show the butterfly diagrams for high- $z$ and low-z groups, respectively.

diagrams, which are constructed using the spectroscopic sample of the COSMOS+AEGIS+XMM-LSS groups at $z<0.6$ (lower panel) and $z>0.6$ (upper panel). The redshift binning is limited by the sample size. In Fig. 15 the filled green and open blue circles show the absolute $r$-band magnitudes of the spectroscopically selected BCGs and satellites as a function of $\Delta M_{1,2}$, respectively. These are quantified by linear regressions (solid black lines), $M_{r, 1}=a_{1}+b_{1} \Delta M_{1,2}$ and $M_{r, 2}=a_{2}+b_{2} \Delta M_{1,2}$. We have compared the results of these linear fits with those for the red-sequence selected BCGs and satellites (dashed red lines). Coefficients of fits are presented in Table 3. This comparison shows that the slopes of the red-sequence selection are slightly shallower than those for the spectroscopic membership and the membership contamination is a little more effective for the high- $z$ groups compared to the low $-z$ groups. However, the trends in the $M_{r, 1}-\Delta M_{1,2}$ and $M_{r, 2}-\Delta M_{1,2}$ relations for both redsequence and spectroscopic members at $z<0.6$ and $z>0.6$ are similar and differences between the slopes/intercepts lie within the corresponding uncertainties. Thus, we expect that our findings which are derived from butterfly diagrams of our XMMLSS catalog are valid within observational errors.

In Fig. 16, we compare the observed $M_{r, 1}-\Delta M_{1,2}$ and $M_{r, 2}-$ $\Delta M_{1,2}$ relations with model predictions of G11 (top panel), B06
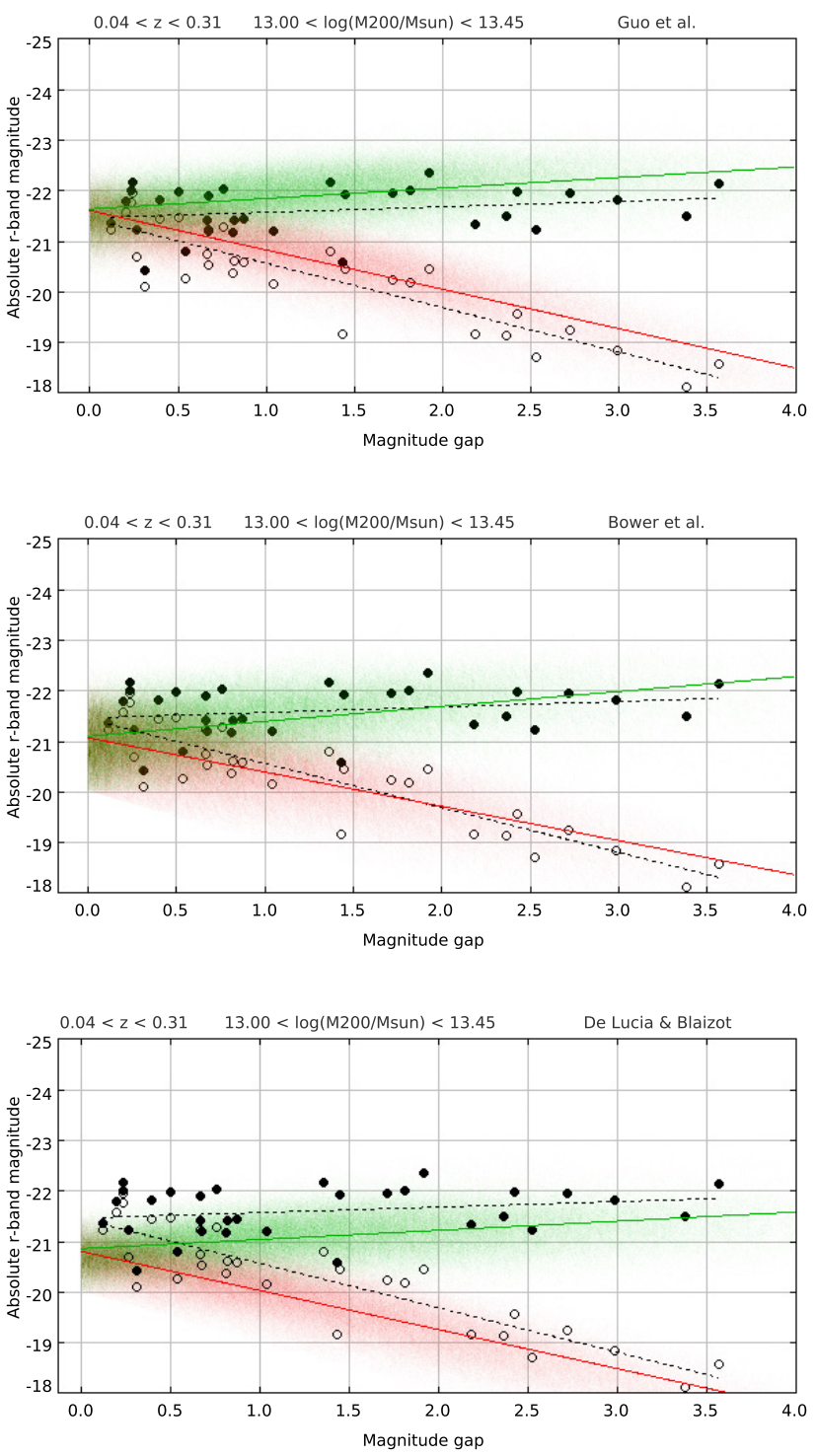

Fig. 16. Absolute $r$-band magnitudes of the first and the second brightest galaxies as a function of the magnitude gap. The S-I data are shown by filled (BCG) and open (2nd-ranked galaxy) black circles with the dashed black lines showing the linear fit. Each panel shows the results of one of the SAMs (G11-top, B06-middle, DLB07-bottom), with green (red) points and a line showing the individual points and the fit to the BCG (2nd-ranked galaxy).

(middle panel) and DLB07 (bottom panel) for S-I. The observed luminosity of the first-ranked galaxy (filled black circles) increases very slowly with $\Delta M_{1,2}$, so the difference in the magnitude is due to the second-ranked galaxies (open black circles), which declines from $M_{r} \sim-21.5$ at $\Delta M_{1,2} \sim 0$ to $M_{r} \sim-18$ at $\Delta M_{1,2} \sim 3.5$. The values of the linear fit coefficients are given in Table 3. For S-I, the galaxies in the G11 model are more luminous than the observed galaxies by $\sim 0.3 \mathrm{mag}$. In contrast, galaxies in the B06 and DLB07 models tend to be less luminous than the observed galaxies by $\sim 0.5$ to $0.7 \mathrm{mag}$.

For the S-II, a similar comparison is presented in Fig. 17. The increase in the absolute magnitude of the BCG with $\Delta M_{1,2}$ is $\approx 3$ higher than in S-I, reaching -22.5 at $\Delta M_{1,2} \sim 3.5$. The absolute magnitude of the satellite galaxy declines from $M_{r} \sim-21.5$ at $\Delta M_{1,2} \sim 0$ to $M_{r} \sim-19$ at $\Delta M_{1,2} \sim 3.5$. As in S-I, the galaxies in the G11 model tend to be 0.5 mag more luminous than the observed ones. The B06 model predicts reasonably well the 

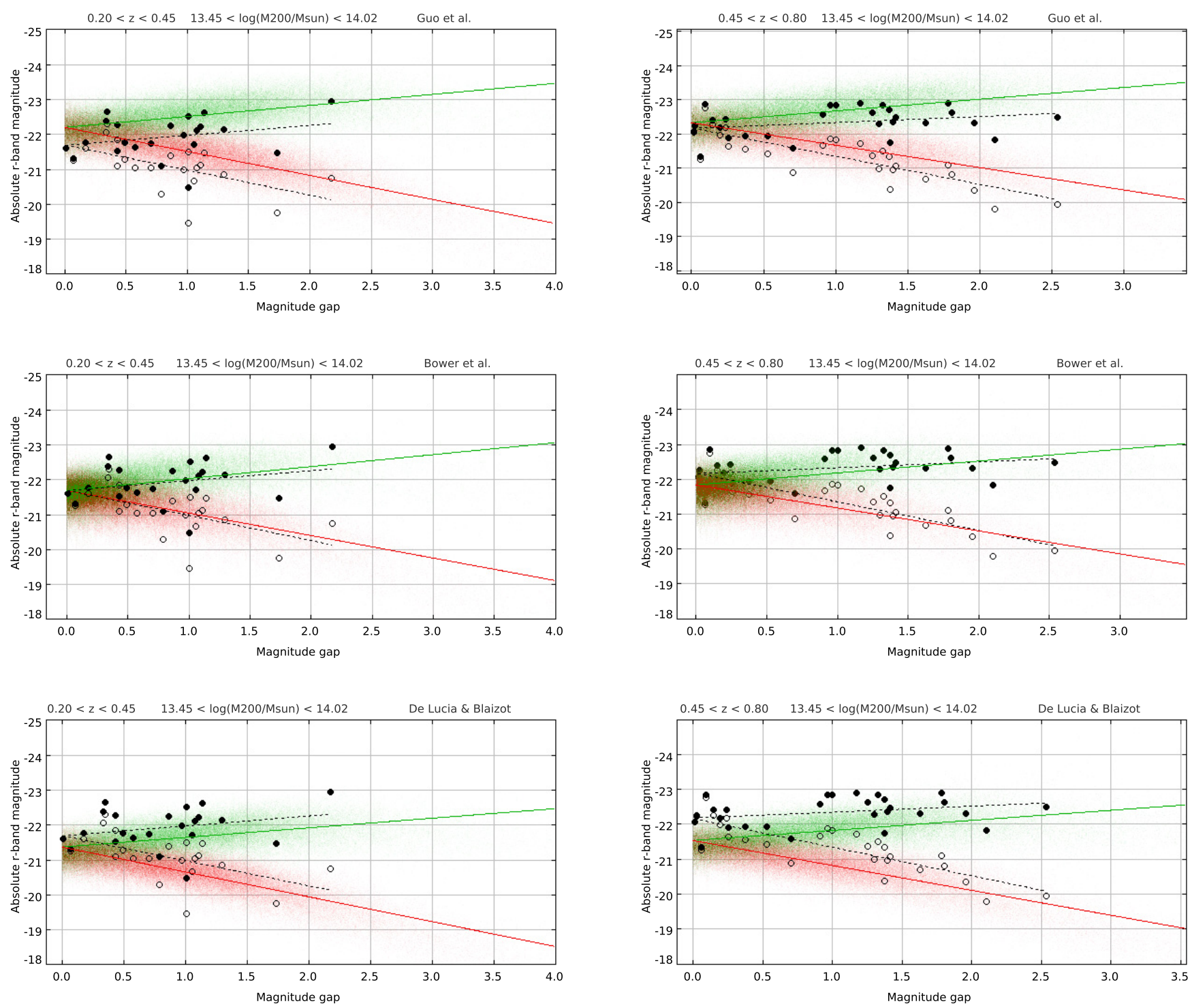

Fig. 17. Same as in Fig. 16, but for the BCGs and second brightest satellites in S-II.

observed luminosity of the first-ranked and second ranked galaxies as a function of $\Delta M_{1,2}$. The predicted luminosities of galaxies in the DLB07 model are less luminous than the observed ones.

We show the butterfly diagrams for S-III in Fig. 18. As for the low- $z$ subsamples, the luminosity of the BCG rises very slowly with $\Delta M_{1,2}$, spanning a range of $-23 \lesssim M_{r} \lesssim-21$ with $\Delta M_{1,2}$. While, the luminosity of the satellite galaxy decreases from $M_{r} \sim-22$ at $\Delta M_{1,2} \sim 0$ to $M_{r} \sim-19$ at $\Delta M_{1,2} \sim 3.5$.

Finally, Fig. 19 illustrates the luminosities of BCG and 2-nd ranked galaxy as a function of magnitude gap for S-IV. The BCG absolute magnitudes span a range of $-24 \lesssim M_{r} \lesssim-21.5$ and increase slowly with $\Delta M_{1,2}$. The luminosity of the second ranked galaxy declines from $M_{r} \sim-22.5$ at $\Delta M_{1,2} \sim 0$ to $M_{r} \sim-20$ at $\Delta M_{1,2} \sim 3.5$.

\subsubsection{Redshift evolution of the butterfly diagram}

In Fig. 20, we compare the redshift evolution of the intercept of $M_{r, 1}-\Delta M_{1,2}$ (top panel) and $M_{r, 2}-\Delta M_{1,2}$ (bottom panel) relations in our observations and the models. We find a significant negative redshift evolution of the intercepts for BCGs and their

Fig. 18. Same as in Fig. 16, but for the BCGs and second brightest satellites in S-III.

satellites by $\sim 0.8$ mag for clusters at $z \gtrsim 0.25$. All models exhibit a shallower evolution of the intercepts with redshift compared to the data. A steeper negative evolution in the BCG magnitudes in time, is most likely due to younger stellar population age of BCGs, compared to the models. The intercept of the predicted butterfly diagram for S-I at low redshifts tend to be closer to G11 compared to two other models, indicating importance of their advances in the modeling. A possible reason for over-prediction of the BCG or BGG luminosity in G11 is that this model incorporates a disruption mechanism which provides additional gas and metal-rich material for the hot gas atmosphere of BCG, thus increasing the cooling rate and star formation in BCG (see G11). To achieve a good agreement with observations, it may still be needed to increase slightly the strength of AGN feedback in this model.

In Fig. 21, we show a lack of the redshift evolution in the slope of the butterfly diagram. No dependence on the absolute magnitude of BCG and an agreement between all models, indicates that the mechanism for creating large gaps is not strongly affected by the star-formation and feedback, and is due to disruption of galaxies. However, as we discussed above, the models 
Table 3. Best linear fit to the $M_{1, r}$ and $M_{2, r}$ as a function of $\Delta M_{1,2}$.

\begin{tabular}{llcccc}
\hline \hline Subsample & Mean redshift & $a 1$ & $b 1$ & $a 2$ & $b 2$ \\
\hline XMM-LSS (our catalog) & & & & & \\
S-I & 0.175 & $-21.44 \pm 0.11$ & $-0.11 \pm 0.10$ & $-21.42 \pm 0.17$ & $0.88 \pm 0.11$ \\
S-II & 0.325 & $-21.66 \pm 0.22$ & $-0.28 \pm 0.15$ & $-21.67 \pm 0.22$ & $0.72 \pm 0.20$ \\
S-III & 0.625 & $-22.13 \pm 0.15$ & $-0.19 \pm 0.12$ & $-22.14 \pm 0.15$ & $0.82 \pm 0.13$ \\
S-IV & 0.95 & $-22.35 \pm 0.15$ & $-0.30 \pm 0.08$ & $-22.35 \pm 0.16$ & $0.70 \pm 0.16$ \\
COSMOS+AEGIS+XMM-LSS & & & & & \\
red-sequence selection & 0.3 & $-22.67 \pm 0.15$ & $-0.10 \pm 0.10$ & $-22.28 \pm 0.14$ & $0.46 \pm 0.10$ \\
spectroscopic selection & 0.3 & $-22.72 \pm 0.14$ & $-0.05 \pm 0.09$ & $-22.34 \pm 0.14$ & $0.53 \pm 0.09$ \\
red-sequence selection & 0.9 & $-23.67 \pm 0.23$ & $0.16 \pm 0.34$ & $-23.69 \pm 0.23$ & $1.26 \pm 0.33$ \\
spectroscopic selection & 0.9 & $-23.86 \pm 0.21$ & $0.37 \pm 0.26$ & $-23.83 \pm 0.20$ & $1.48 \pm 0.25$ \\
\hline
\end{tabular}

Notes. The four upper rows present coefficients of the linear fit to the butterfly diagrams of our catalog and four lower rows list those for the sample of 84 groups from COSMOS+AEGIS+XMM-LSS
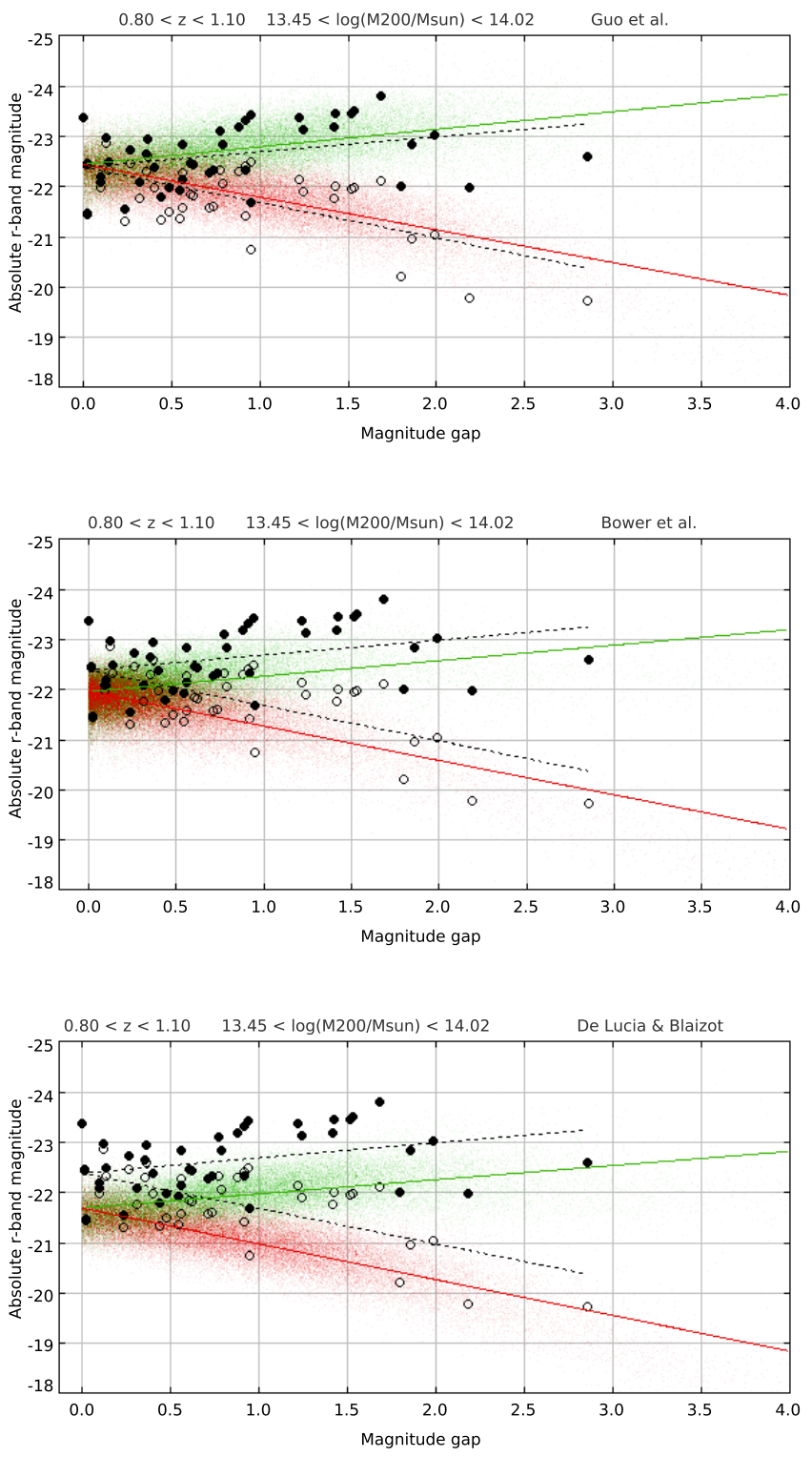

Fig. 19. Same as in Fig. 16, but for the BCGs and the second brightest satellites in S-IV.

show differences in the fraction of high gap systems, which is controlled by the efficiency of the transformation.
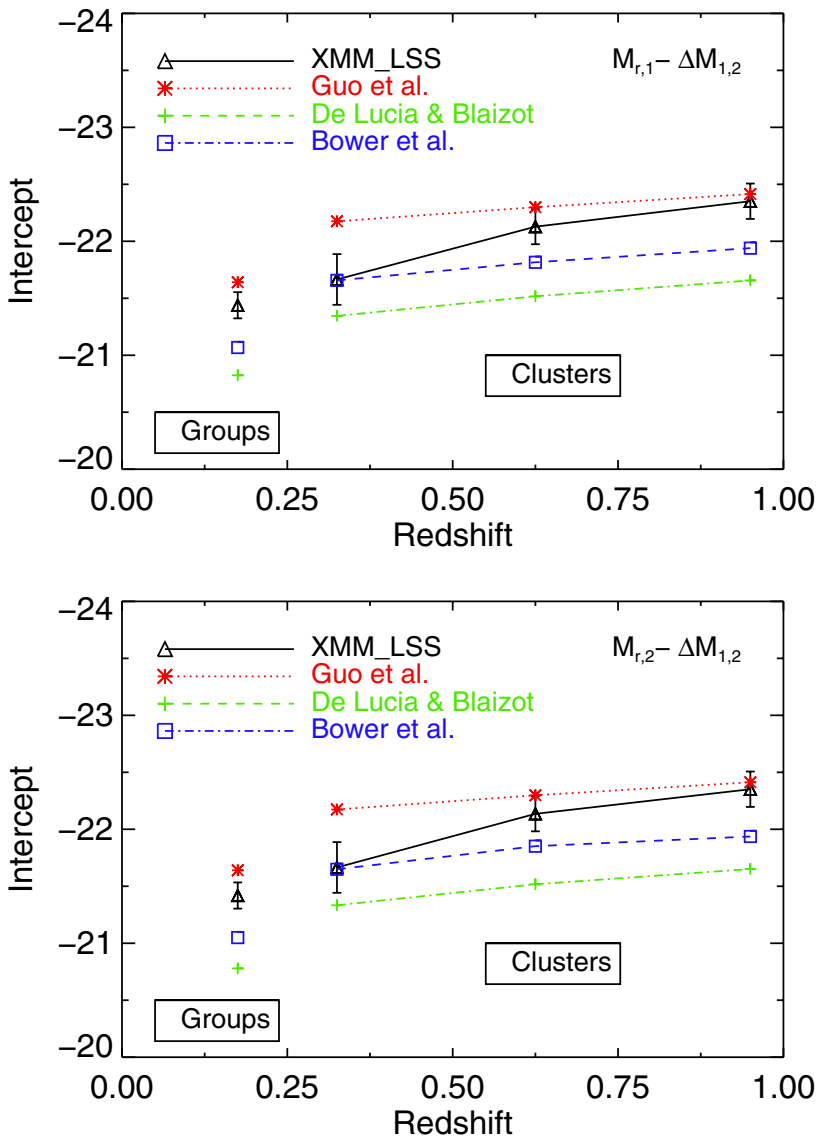

Fig. 20. Evolution of the zero point of the $M_{r, 1}-\Delta M_{1,2}$ (upper panel.) and $M_{r, 2}-\Delta M_{1,2}$ (bottom panel.) relations with redshift. The solid black curve with error bars represents the data. Dotted red, dashed blue and dash-dotted green curves show the model predictions of G11, B06 and DLB07, respectively. In contrast to the models, we observe a significant evolution with redshift.

\subsection{Fossil groups}

Using the magnitude gap, we identify 23 fossil groups in our catalog. The fossil groups constitute $22.2 \pm 6 \%$ and $12.3 \pm 7.0 \%$ of all identified X-ray groups in our catalog at $z \leq 0.6$ and $z>0.6$, respectively. In this calculation we include the impact of the contamination (when using the red-sequence method versus the spectroscopic approach to select group membership) and completeness effects on the gap measurements. This misclassification causes the fraction of fossil groups to be underestimated by $\pm 20 \%$ at high- $z$, which we add to the errors. Although the 

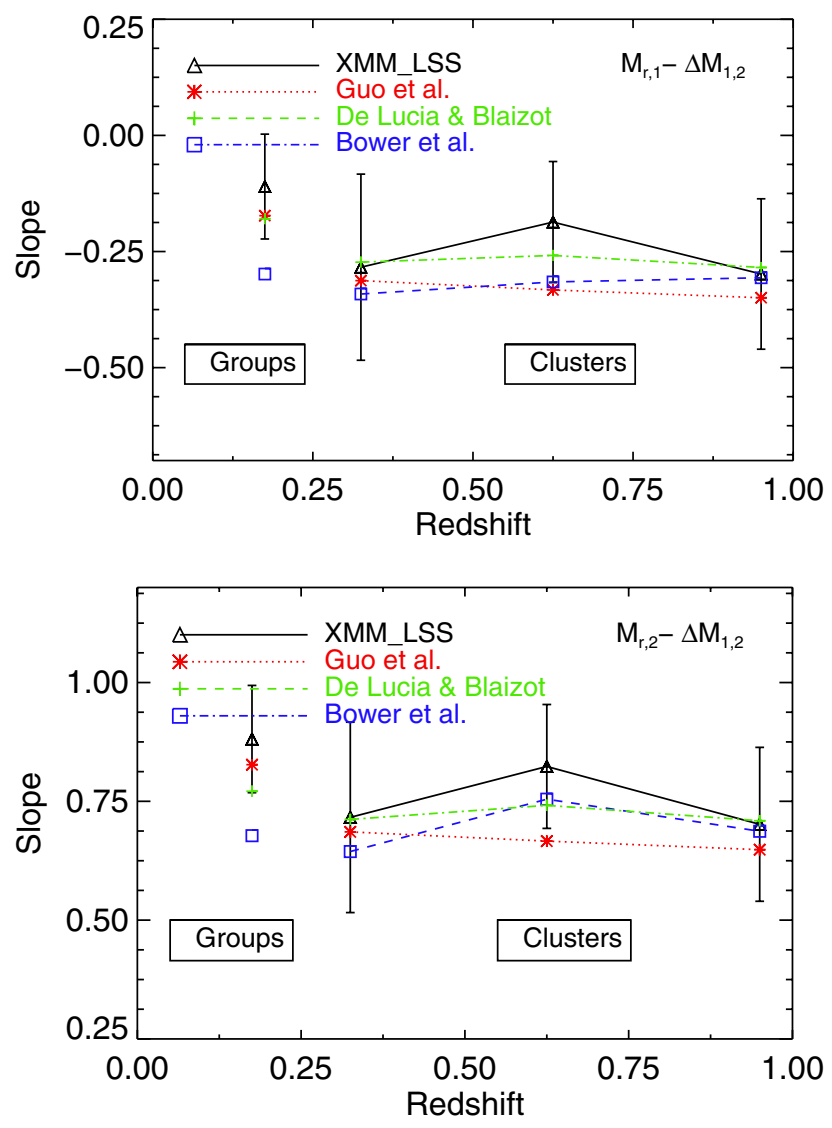

Fig. 21. Evolution of the slopes of the $M_{r, 1}-\Delta M_{1,2}$ (upper panel) and $M_{r, 2}-\Delta M_{1,2}$ (bottom panel) relations with redshift. The models and the data reveal a nearly constant evolution with redshift.

significance of the redshift evolution of the fraction of the fossil groups is marginal, it is also found in a nearly independent sample of the COSMOS+AEGIS+XMM-LSS groups, which contains $23.1 \pm 7 \%$ and $10.5 \pm 8 \%$ of all groups as fossil groups at $z \leq 0.6$ and $z>0.6$, respectively.

We mark the fossil groups in Fig. 7 with red circles to illustrate their occurrence as a function of total mass and redshift. Given the previous results on the prevalence of cool cores among the fossil groups, in the following we verify that we do not have a preferential selection of the fossil groups. Given the limited statistics of the X-ray data, we select the extent of X-ray detection as a parameter of the comparison. If the extends are much smaller, it would indicate that the central part of the emission dominates the detection and the X-ray selection of fossils is different to the bulk of the groups.

We compare the distributions of $\frac{R_{\mathrm{X}}}{R_{500}}$ ratio for fossils to nonfossils, selected by implying $\Delta M_{1,2} \leq 0.5$ in Fig. 22 (top panel). $R_{\mathrm{X}}$ is the detected extent of the $\mathrm{X}$-ray emission, while $R_{500}$ is the extent of $\mathrm{X}$-ray emission anticipated for the given flux of the group. The distribution of $\frac{R_{\mathrm{X}}}{R_{500}}$ ratio is skewed toward the lower values for fossils compared to the non-fossil groups, with $1 \%$ probability of chance occurrence, based on the K-S test. However, as also shown in Fig. 22, the observed extent of the $\mathrm{X}$-ray emission is a strong function of the redshift of the group. So, for a refined comparison of the extent of X-ray detection for fossil candidates and non-fossils, we separate the groups into two redshift bins: with $z>0.6$ and $z \leq 0.6$. We find that fossils exhibit only a marginally lower values of $\frac{R_{X}}{R_{500}}$ ratio compared to non-fossils in both redshift ranges, $z>0.6$ and $z \leq 0.6$, which may also be due to residual differences in the redshift
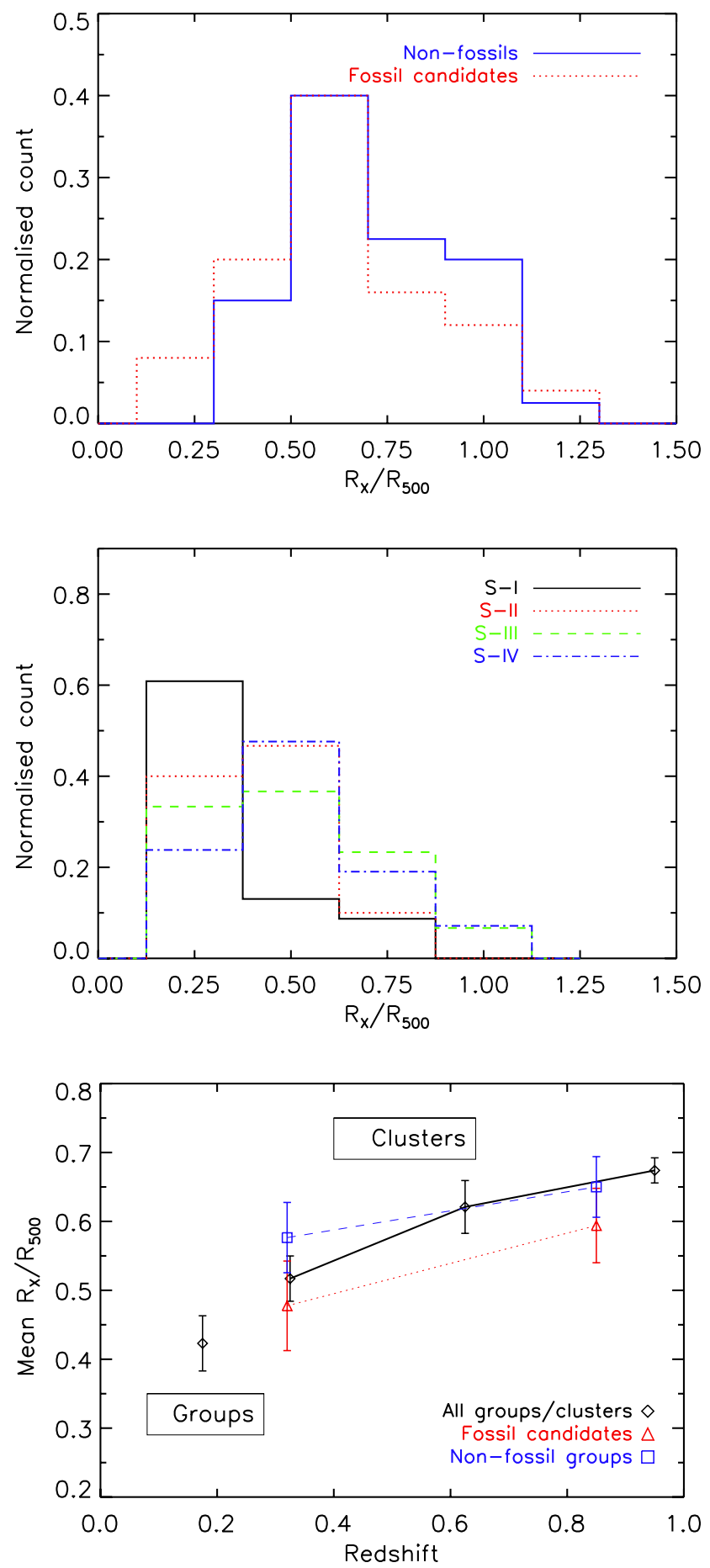

Fig. 22. Upper panel. Distribution of the ratio of the X-ray extent of the detection to the total extent of X-ray emission, $\frac{R_{\mathrm{X}}}{R_{500}}$ for the fossil group candidates $\left(\Delta M_{1,2} \geq 1.7\right)$ (dotted blue histogram) and non-fossils $\left(\Delta M_{1,2} \leq 0.5\right)$ (solid red histogram) in our X-ray group catalog. The fraction of fossil groups is skewed toward the lower values of $\frac{R_{\mathrm{X}}}{R_{500}}$ compared to the non-fossil groups. Middle panel. The $\frac{R_{\mathrm{X}}}{R_{500}}$ distribution for $\mathrm{X}$-ray groups in different subsamples. Lower panel. The redshift evolution of the extent of X-ray detection for galaxy groups/clusters. A redshift evolution in the ratio by 0.25 in amplitude is seen for all groups.

distribution. In our method, the cores of groups are removed prior to detection, which as this test shows, reduces the impact of X-ray selection. 
In Fig. A.1 to Fig. A.23 we show the X-ray emission as contours on the CFHTLS RGB images for the fossils candidate groups listed in Table A.1. We show the color magnitude diagrams of $g^{\prime}-r^{\prime}$ and $r^{\prime}-i^{\prime}$ versus $z^{\prime}$ for the fossil candidates at $z<0.66$, and $r^{\prime}-i^{\prime}$ and $i^{\prime}-z^{\prime}$ versus $z^{\prime}$ for those at $z \geq 0.66$. Group members (dark circles) are selected according to the method described in Sect. 4.1. We mark the BGG and the second brightest galaxies on each RGB image and all color magnitude diagrams.

\section{Summary and conclusions}

We search for the extended X-ray emission using the contiguous XMM coverage of the CFHTLS W1 field by the XMMLSS public data. We provide a catalog of 129 X-ray galaxy groups including 45 groups with a spectroscopic redshift over $\sim 3$ degrees $^{2}$, spanning the redshift range $0.04<z<1.23$, characterised by a rest frame $0.1-2.4 \mathrm{keV}$ band luminosity range between $10^{41}$ and $10^{44} \mathrm{ergs} \mathrm{s}^{-1}$. We utilize a two-color redsequence finder for the photometric selection of group members and to calculate the mean group redshift. For the first time, we perform a statistical analysis on the luminosity gap of groups over the wide redshift range of our catalog. We compare our observational results with predictions of SAMs presented in G11, DLB07 and B06. Our main results are as follows:

(i) We have compared the flux for the groups and clusters in common with the $\mathrm{C} 1$ clusters of Adami et al. (2011). We show that clusters at $z>0.6$ have important differences in flux estimates as a consequence of point source contamination.

(ii) We show that two-color selection of group members reduces the sample contamination by DSFGs at $z \lesssim 0.6$ compared to a single color selection. This contamination slightly affects the sample at $z \gtrsim 0.6$ (by $\approx 3 \%$ ).

In addition, to test the effect of a red sequence selection of members on the magnitude gap measurements and the determination of the fraction of fossil or non-fossil groups, we compare the magnitude gap values computed using the spectroscopic and red sequence members for a sample of 84 spectroscopic X-ray groups from our catalogs in the AEGIS, COSMOS and XMM-LSS fields. We find that the systematic effect on the magnitude gap computation is limited to $<6 \%$ at $95 \%$ Poisson confidence level, which is low compared to our statistical uncertainties. We apply this effect on our estimate of the fraction of fossil groups and number of groups in each $\Delta M_{1,2}$ bin in Fig. 12. Using this sample we show that our results which are derived by combining groups with redsequence and spectroscopic membership are valid and we quantified the corresponding uncertainties.

(iii) We demonstrate that the fraction of groups as a function of $\Delta M_{1,2}$ exhibits a flatter slope for low- $z$ and low-mass groups in S-I compared to higher redshift subsamples, revealing the build-up of high magnitude gap groups at low redshift. We derive a marginally higher fraction of groups with $\Delta M_{1,2} \geq 2$ compared to all models for S-I. We find that the B06 model is not able to reproduce the observed trends for $\Delta M_{1,2}$ distributions. On the other hand, G11 and DLB07 models predict reasonably well $\Delta M_{1,2}$ distributions for groups and clusters. It appears that the magnitude gap distribution of groups with $\Delta M_{1,2} \lesssim 2$ is well modeled by these two models. However the consistency between them and observations is reduced for groups and clusters with large magnitude gap, such as fossil groups, indicating that the fraction of these objects have not been understood completely. We report a good agreement between distributions of the magnitude gap for our groups catalog with red sequence selection of group members and that of our spectroscopic sample of 84 groups within the redshift range of $0.05<z<1.22$ (see Fig. 11).

(iv) We show that the model abundance by volume of galaxy groups is in agreement with low- $z$ X-ray groups in S-I. However, at higher redshifts the observed number density of groups with higher halo masses is below the predicted model values which we attribute to cosmic variance and sensitivity of the abundance of high-mass systems to cosmological parameters. We demonstrate that the observed mean fraction of $\mathrm{X}$-ray clusters with $\Delta M_{1,2} \geq 1$ evolves slowly with redshift in agreement with all models, with the exception of B06, which under-predicts the fraction of clusters with $\Delta M_{1,2} \geq 1$. A larger fraction of groups with $\Delta M_{1,2} \geq 1$ is detected at lower redshifts, in agreement with the models.

(v) We investigate the absolute $r$-band magnitude of the first and the second brightest galaxies as a function of the magnitude gap. We find a significant negative evolution of the intercept ( $\sim 0.8 \mathrm{mag}$ ) of the $M_{r, 1}-\Delta M_{1,2}$ and $M_{r, 2}-\Delta M_{1,2}$ relations for clusters at $0.2 \lesssim z \lesssim 1.10$. In comparison, all the models predict a flatter redshift evolution for the intercepts. We attribute the steeper negative evolution in the BCG magnitudes to a more recent build-up of stellar mass in these galaxies (in agreement with the models), suggestive of a stronger redshift dependence of AGN feedback. The G11 model predicts well the intercepts for low- $z$ and low-mass groups, indicating the importance of the improvements which have been made to this model. Conversely, B06 and DLB07 models underpredict the intercepts for the BGGs and their satellites. The observed slope of the $M_{r, 1}-\Delta M_{1,2}$ and $M_{r, 2}-\Delta M_{1,2}$ relations for clusters show a nearly constant redshift evolution, consistent with all models. We conclude that the mechanisms for creating magnitude gaps is well modeled in the SAMs. For galaxy groups, we find that the G11 and DLB07 models predict reasonably well the slopes of the butterfly diagrams. We conclude that satellite distribution in galaxy groups is too efficient in the B06 model.

(vi) We have selected 23 fossil group candidates using the magnitude gap criterion. Similar to other studies we find that fossil groups constitute $22.2 \pm 6 \%$ of all groups at $z<0.6$. We report a redshift evolution in this fraction which drops to $12.3 \pm 7 \%$ at $z>0.6$. These numbers and their evolution are consistent with those of our spectroscopic sample of COSMOS+AEGIS+XMM-LSS groups where we classify $23.1 \pm 7 \%$ and $10.5 \pm 8 \%$ of all groups as fossils at $z \leq 0.6$ and $z>0.6$, respectively. We show that some fossil group candidates include few galaxies, located outside the $0.5 R_{200}$, which are brighter than the second bright galaxy which used for the magnitude gap estimate, supporting a suggestion of Von Benda-Beckmann et al. (2008) that large magnitude gaps can be refilled by infall.

Finally, this study demonstrates that magnitude gap can be used to differentiate between models. We find that the recent improvements in the SAM of G11 brings it closer to the observations compared to the early version of the SAMs, DBL07 and B06. However, it still fails to reproduce all the results. For current SAMs only a single model output is available. However, in order to improve the understanding further, a grid of model predictions fully sampling the parameter space of amplitude and redshift evolution of the relevant physical mechanisms such as AGN feedback is essential. 
Acknowledgements. Initial stages of this work have been partially funded by the German Deutsche Forschungsgemeinschaft, DFG Leibniz Prize (FKZHA1850/28 - 1). This work also has been partially supported by the grant of Finish Academy of Sciences to the University of Helsinki, decision number 266918. We thank Guenther Hasinger, Hannu Koskinen, Kirpal Nandra, Christophe Adami, Birgit Boller, Marguerite Pierre, Peter Johansson and Kimmo Kettula for helpful assistance and comments. We thank the anonymous referee for the patience in reviewing the manuscript and many insightful comments. We used the Millennium Simulation databases in this paper and the web application providing online access to them were constructed as part of the activities of the German Astrophysical Virtual Observatory.

\section{References}

Adami, C., Mazure, A., Pierre, M., et al. 2011, A\&A, 526, A18

Aihara, H., Allende, P. C., An, D., et al. 2011, ApJS, 193, 29

Allevato, V., Finoguenov, A., Hasinger, G., et al. 2012, ApJ, 758, 47

Arnouts, S., Moscardini, L., Vanzella, E., et al. 2002, MNRAS, 329, 355

Barnes, J. E. 1989, Nature, 338, 123

Bauer, F. E., Alexander, D. M., Brandt, W. N., et al. 2002, AJ, 123, 1163

Benson, A. J., Bower, R. G., Frenk, C. S., et al. 2003, ApJ, 599, 38

Bekki, K., Forbes, D. A., Beasley, M. A., \& Couch, W. J. 2002, MNRAS, 335, 1176

Bielby, R. M., Finoguenov, A., Tanaka, M., et al. 2010, A\&A, 523, A66 Bordoloi, R., Lilly, S. J., Amara, A., et al. 2012, MNRAS, 421, 1671 Borgani, S., \& Guzzo, L. 2001, A\&A, 409, 39

Bower, R. G., Benson, A. J., Malbon, R., et al. 2006, MNRAS, 370, 645

Brimioulle, F., Lerchster, M., Seitz, S., Bender, R., \& Snigula, J. 2008 [arXiv:0811.3211]

Brimioulle, F., Seitz, S., Lerchster, M., Seitz, S., Bender, R., \& Snigula, J. 2013, MNRAS, 432, 1046

Bruzual, G., \& Charlot, S. 2003, MNRAS, 344, 1000

Calzetti, D., Armus, L., Bohlin, R. C., et al. 2000, ApJ, 533, 682

Cole, S., Lacey, C. G., Baugh, C. M., \& Frenk, C. S. 2000, MNRAS, 319, 168

Chabrier, G. 2003, PASP, 115, 763

Colless, M., Dalton, G., Maddox, S., et al. 2001, MNRAS, 328, 1039

Connelly, J. L., Wilman, D. J., Finoguenov, A., et al. 2012, ApJ, 756, 139

Conroy, C., Ho, S., \& White, M. 2007a, MNRAS, 379, 1491

Conroy, C., Wechsler, R. H., \& Kravtsov, A. V. 2007b, ApJ, 668, 826

Croton, D. J., Springel, V., White, S. D. M., et al. 2006, MNRAS, 365, 11

D’Onghia, E., Sommer-Larsen, J., Romeo, A. D., et al. 2005, ApJ, 630, L109

Dariush, A. A., Khosroshahi, H. G., Ponman, T. J., et al. 2007, MNRAS, 382, 433

Dariush, A. A., Raychaudhury, S., Ponman, T. J., et al. 2010, MNRAS, 405, 1873

Davis, M., Efstathiou, G., Frenk, C. S., \& White, S. D. M., 1985, ApJ, 292, 371 De Lucia, G., \& Blaizot, J. 2007, MNRAS, 375, 2

De Lucia, G., Springel, V., White, S. D. M., Croton, D., \& Kauffmann, G. 2006, MNRAS, 366, 499

Díaz-Giménez, E., Zandivarez, A., Proctor, R., Mendes de Oliveira, C., \& Abramo, L. R. 2011, A\&A, 527, A129

Eigenthaler, P., \& Zeilinger, W. W. 2013, A\&A, 553, A99

Erben, T., Hildebrandt, H., Miller, L., et al. 2013, MNRAS, 433, 2545

Erfanianfar, G., Finoguenov, A., Tanaka, M., et al. 2013, ApJ, 765, 117

Erfanianfar, G., Popesso, P., Finoguenov, A., et al. 2014, MNRAS, submitted

Fioc, M., \& Rocca-Volmerange, B. 1999 [arXiv: astro-ph/9912179]

Finoguenov, A., Streblyanska, A., Hasinger, G., Hashimoto, Y., \& Szokoly, G. 2005, Adv. Space Res., 36, 710

Finoguenov, A., Guzzo, L., Hasinger, G., et al. 2007, ApJS, 172, 182

Finoguenov, A., Connelly, J. L., Parker, L. C., et al. 2009, ApJ, 704, 564

Finoguenov, A., Watson, M. G., Tanaka, M., et al. 2010, MNRAS, 403, 2063

Font, A. S., Bower, R. G., McCarthy, I. G., et al. 2008, MNRAS, 389, 1619

George, M. R., Leauthaud, A., Bundy, K., et al. 2011, ApJ, 742, 125

Giacconi, R., Zirm, A., Wang, J., et al. 2002, ApJS, 139, 369

Giodini, S., Pierini, D., Finoguenov, A., et al. 2009, ApJ, 703, 982

Guo, Q., White, S., Boylan-Kolchin, M., et al. 2011, MNRAS, 413, 101

Harker, G., Cole, S., Helly, J., Frenk, C., \& Jenkins, A. 2006, MNRAS, 367, 1039

Hearin, A. P., Zentner, A. R., Berlind, A. A., \& Newman, J. A. 2013, MNRAS, 433,659

Henriques, B. M. B., \& Thomas, P. A. 2010, MNRAS, 403, 768
Humphrey, P. J., Buote, D. A., Brighenti, F., et al. 2012, ApJ, 748, 11 Huntley, J. M. 1980, ApJ, 238, 524

Ilbert, O., Arnouts, S., McCracken, H. J., et al. 2006, A\&A, 457, 841

Ilbert, O., Capak, P., Salvato, M., et al. 2009, ApJ, 690, 1236

Jansen, F., Lumb, D., Altieri, B., et al. 2001, A\&A, 365, L1

Jones, L. R., Ponman, T. J., Horton, A., et al. 2003, MNRAS, 343, 627

Junqueira, T. C., Lépine, J. R. D., Braga, C. A. S., \& Barros, D. A. 2013, A\&A, 550, A91

Kauffmann, G., \& Haehnelt, M. 2000, MNRAS, 311, 576

Kennicutt, R. C., Jr. 1983, ApJ, 272, 54

Khosroshahi, H. G., Maughan, B. J., Ponman, T. J., \& Jones, L. R. 2006, MNRAS, 369, 1211

Khosroshahi, H. G., Ponman, T. J., \& Jones, L. R. 2007, MNRAS, 377, 595

Kim, H.-S., Baugh, C. M., Cole, S., Frenk, C. S., \& Benson, A. J. 2009, MNRAS, 400,1527

La Barbera, F., Paolillo, M., De Filippis, E., \& de Carvalho, R. R. 2012, MNRAS, 422,3010

Leauthaud, A., Finoguenov, A., Kneib, J.-P., et al. 2010, ApJ, 709, 97

Le Fèvre, O., Vettolani, G., Paltani, S., et al. 2004, A\&A, 428, 1043

Le Fèvre, O., Vettolani, G., Garilli, B., et al. 2005, A\&A, 439, 845

Libeskind, N. I., Cole, S., Frenk, C. S., \& Helly, J. C. 2006, MNRAS, 368, 1381

Liu, L., Yang, X., Mo, H. J., van den Bosch, F. C., \& Springel, V. 2010, ApJ, 712,734

Maraston, C., Strömbäck, G., Thomas, D., Wake, D. A., \& Nichol, R. C. 2009, MNRAS, 394, L107

Milosavljević, M., Miller, C. J., Furlanetto, S. R., \& Cooray, A. 2006, ApJ, 637, L9

Mirkazemi, M., Finoguenov, A. Pereira, M. J., et al. 2014, ApJ, submitted

Mo, H. J., Mao, S., \& White, S. D. M. 1998, MNRAS, 295, 319

Pacaud, F., Pierre, M., Refregier, A., et al. 2006, MNRAS, 372, 578

Pacaud, F., Pierre, M., Adami, C., et al. 2007, MNRAS, 382, 1289

Papovich, C., Momcheva, I., Willmer, C. N. A., et al. 2010, ApJ, 716, 1503

Percival, W. J., Reid, B. A., Eisenstein, D. J., et al. 2010, MNRAS, 401, 2148

Pierre, M., Chiappetti, L., Pacaud, F., et al. 2007, MNRAS, 382, 279

Pierre, M., Clerc, N., Maughan, B., et al. 2012, A\&A, 540, A4

Ponman, T. J., Allan, D. J., Jones, L. R., Merrifield, M., \& MacHardy, I. M. 1994, Nature, 369, 462

Press, W. H., \& Schechter, P. 1974, ApJ, 187, 425

Rosati, P., Borgani, S., \& Norman, C. 2002, ARA\&A, 40, 539

Salvato, M., Hasinger, G., Ilbert, O., et al. 2009, ApJ, 690, 1250

Scoville, N., Aussel, H., Brusa, M., et al. 2007, ApJS, 172, 1

Silverman, J. D., Kovač, K., Knobel, C., et al. 2009, ApJ, 695, 171

Smith, G. P., Khosroshahi, H. G., Dariush, A., et al. 2010, MNRAS, 409, 169

Somerville, R. S., Primack, J. R., \& Faber, S. M. 2001, MNRAS, 320, 504

Spergel, D. N., Verde, L., Peiris, H. V., Komatsu, E., et al. 2003, ApJS, 148, 175

Springel, V., Yoshida, N., \& White, S. D. M. 2001, New Astron., 6, 79

Springel, V., White, S. D. M., Jenkins, A., et al. 2005, Nature, 435, 629

Strüder, L., Briel, U., Dennerl, K., et al. 2001, A\&A, 365, 18

Su, Y., White, R. E., III, \& Miller, E. D. 2013, ApJ, 775, 89

Šuhada, R., Song, J., Böhringer, H., et al. 2012, A\&A, 537 , A39

Tanaka, M., Finoguenov, A., Kodama, T., et al. 2008, A\&A, 489, 571

Tanaka, M., Finoguenov, A., \& Ueda, Y. 2010, ApJ, 716, L152

Tanaka, M., Finoguenov, A., Lilly, S. J., et al. 2012, PASJ, 64, 22

Tavasoli, S., Khosroshahi, H. G., Koohpaee, A., Rahmani, H., \& Ghanbari, J. 2011, PASP, 123, 1

Turner, M. J. L., Abbey, A., Arnaud, M., et al. 2001, A\&A, 365, L27

van den Bosch, F. C., Yang, X., Mo, H.J., et al. 2007, MNRAS, 376, 841

van den Bosch, F. C., More, S., Cacciato, M., Mo, H., \& Yang, X. 2013, MNRAS, 430,725

Voevodkin, A., Borozdin, K., Heitmann, K., et al. 2010, ApJ, 708, 1376

Von Benda-Beckmann, A. M., D’Onghia, E., Gottlöber, S., et al. 2008, MNRAS, 386,2345

Weinberg, S. 1972, Gravitation and Cosmology: Principles and Applications of the General Theory of Relativity (Wiley-VCH)

White, S. D. M., \& Frenk, C. S. 1991, ApJ, 379, 52

White, S. D. M., \& Rees, M. J. 1978, MNRAS, 183, 341

Yang, X., Mo, H., \& van den Bosch, F. 2008, ApJ, 676, 248

Yang, X., Mo, H. J., Jing, Y. P., \& van den Bosch, F. C. 2005, MNRAS, 358, 217

Yang, X., Mo, H. J., \& van den Bosch, F. C. 2009, ApJ, 695, 900

Zandivarez, A., Martínez, H. J., \& Merchán, M. E. 2006, ApJ, 650, 137

Zel'dovich, Y. B. 1970, A\&A, 5, 84

Zibetti, S., Pierini, D., \& Pratt, G. W. 2009, MNRAS, 392, 525 
G. Gozaliasl et al.: Evolution of the luminosity gap in X-ray galaxy groups

Table A.1. Fossil group candidates.

\begin{tabular}{|c|c|c|c|c|c|}
\hline $\begin{array}{c}\text { Group ID } \\
\text { (1) } \\
\end{array}$ & $\begin{array}{l}z_{\text {phot }} \\
\text { (2) }\end{array}$ & $\begin{array}{c}\mathrm{RA}_{\mathrm{BGG}}(\mathrm{J} 2000) \\
(3)\end{array}$ & $\begin{array}{c}\operatorname{Dec}_{\mathrm{BGG}}(\mathrm{J} 2000) \\
(4)\end{array}$ & $\begin{array}{l}r^{\prime} \\
(5) \\
\end{array}$ & $\begin{array}{c}\Delta M_{1,2} \\
(6)\end{array}$ \\
\hline 101540 & 0.07 & 36.3736 & -4.0120 & 14.219 & 1.922 \\
\hline 101391 & 0.07 & 36.1958 & -4.1475 & 16.929 & 3.297 \\
\hline 102820 & 0.11 & 36.9687 & -3.8552 & 17.213 & 2.059 \\
\hline 100130 & 0.13 & 36.8133 & -5.0756 & 16.367 & 2.645 \\
\hline 103330 & 0.14 & 35.3946 & -4.038 & 17.142 & 6.922 \\
\hline 101210 & 0.15 & 36.3319 & -4.317 & 16.634 & 1.747 \\
\hline 100150 & 0.16 & 35.2821 & -4.9992 & 17.302 & 2.957 \\
\hline 100010 & 0.22 & 36.4159 & -5.0052 & 17.241 & 1.714 \\
\hline 102130 & 0.23 & 35.6247 & -3.8769 & 18.470 & 2.232 \\
\hline 101290 & 0.25 & 36.6974 & -4.2531 & 18.733 & 2.514 \\
\hline 101480 & 0.34 & 36.6486 & -4.0695 & 18.025 & 2.157 \\
\hline 100640 & 0.34 & 35.5052 & -4.6332 & 18.128 & 2.022 \\
\hline 101200 & 0.47 & 35.6688 & -4.3064 & 20.393 & 4.607 \\
\hline 103320 & 0.53 & 36.5861 & -3.7339 & 20.354 & 1.791 \\
\hline 101730 & 0.6 & 36.0241 & -3.9213 & 20.130 & 1.955 \\
\hline 101360 & 0.6 & 36.8554 & -4.1823 & 20.489 & 2.728 \\
\hline 100620 & 0.66 & 37.1107 & -4.6587 & 22.320 & 2.103 \\
\hline 101000 & 0.76 & 35.8876 & -4.4227 & 21.136 & 1.727 \\
\hline 102220 & 0.86 & 35.7790 & -3.7906 & 22.957 & 2.187 \\
\hline 100120 & 0.88 & 35.3644 & -5.067 & 22.125 & 3.080 \\
\hline 101490 & 0.94 & 35.3728 & -4.0977 & 22.336 & 1.989 \\
\hline 100430 & 0.98 & 36.1239 & -4.8010 & 22.342 & 1.861 \\
\hline 101120 & 1.1 & 35.5422 & -4.3494 & 21.181 & 2.808 \\
\hline
\end{tabular}

\section{Appendix A: Fossil group candidates}

In Table A1 we present fossil group candidates catalog. Columns 1 and 2 are group id and the photometric redshift of group. Columns 3 and 4 present the BGG coordinate RA.(J2000) and Dec.(J2000) in degrees. Columns 5 and 6 are BGG $r^{\prime}$-band magnitude and $\Delta M_{1,2}$. In Figs. A.1-A.23 we show the $\mathrm{X}$-ray emission as contours overlaid on the CFHTLS RGB image (top panel) and the color magnitude diagrams of $g^{\prime}-r^{\prime}$ and $r^{\prime}-i^{\prime}$ versus $z^{\prime}$ for $z<0.6$ and $r^{\prime}-i^{\prime}$ and $i^{\prime}-z^{\prime}$ versus $z^{\prime}$ for $z>0.6$ for fossil group candidates listed in Table A.1.

In Sect. 5.2 we define a fossil group and the search radius used for the magnitude gap calculation. Group members selected using two colors according to the method described in Sect. 4.1. We show group members with filled black circles in each color magnitude diagrams. The first and second brightest galaxies are marked by red asterisks. The upper and lower limits of colors in each redshift have been shown with horizontal dotted blue lines.

As it can be seen in the color magnitude diagrams, in some galaxy groups there are galaxies outside the $0.5 R_{200}$ that are brighter than the second galaxy which is used to compute the $\Delta M_{1,2}$. These groups are experiencing the infall of galaxies into search radius. This demonstrates that the magnitude gap estimate and the identification of fossil groups are very sensitive to the adopted search radius for selecting the second bright galaxy.
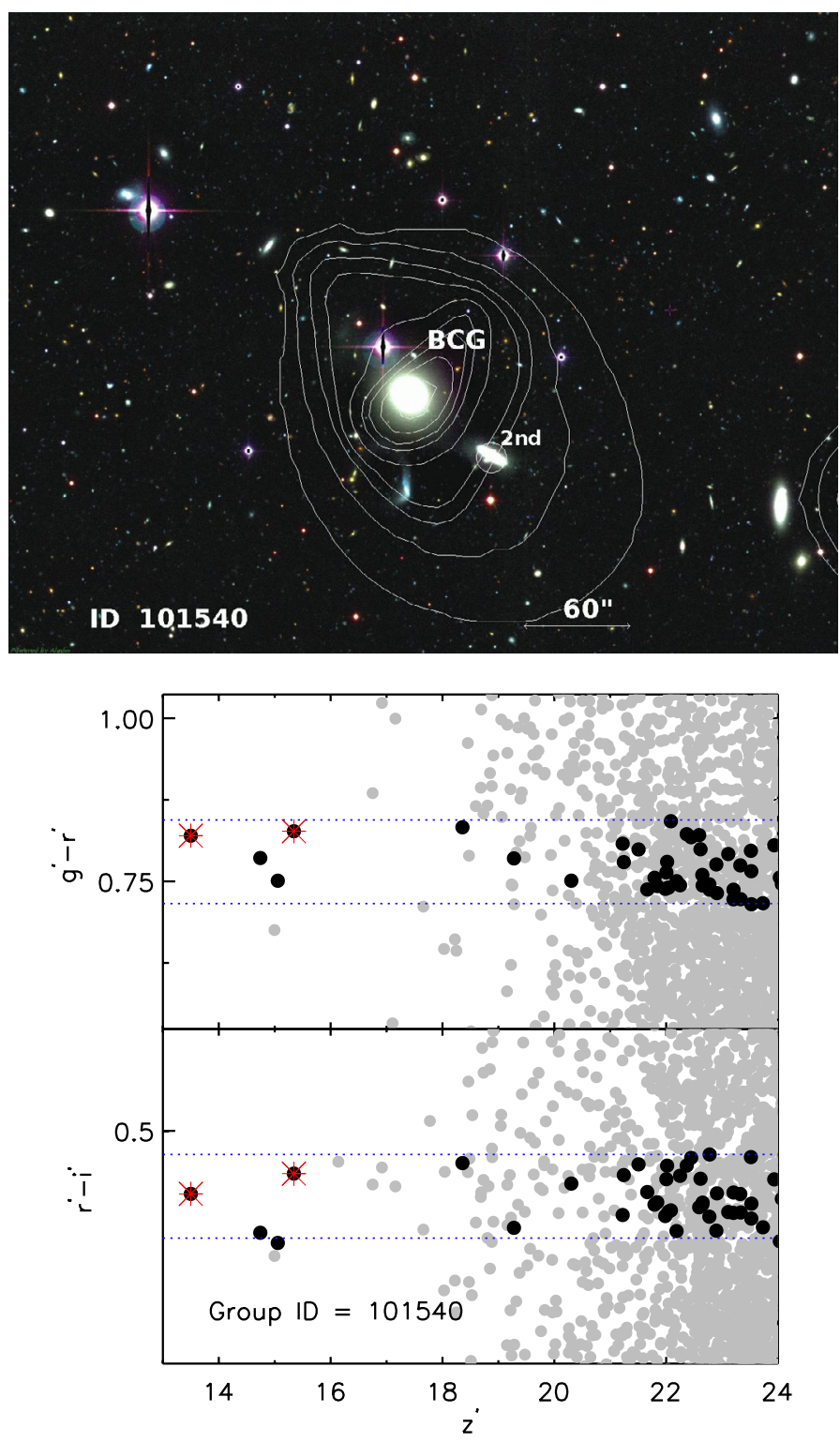

Fig. A.1. Upper panel: contours of the extended X-ray emission overlaid on the CFHTLS RGB image of the fossil group 101540 at $z=0.07$. Middle panel: $g^{\prime}-r^{\prime}$ versus $z^{\prime}$. Lower panel: $r^{\prime}-i^{\prime}$ versus $z^{\prime}$. Filled black circles illustrate group members selected by the method described in Sect. 4.1. The BGG and second brightest satellite galaxy within $0.5 R_{200}$ have been marked with red asterisks within each color magnitude diagram. The upper and lower limits of colors are shown by horizontal dashed lines. 

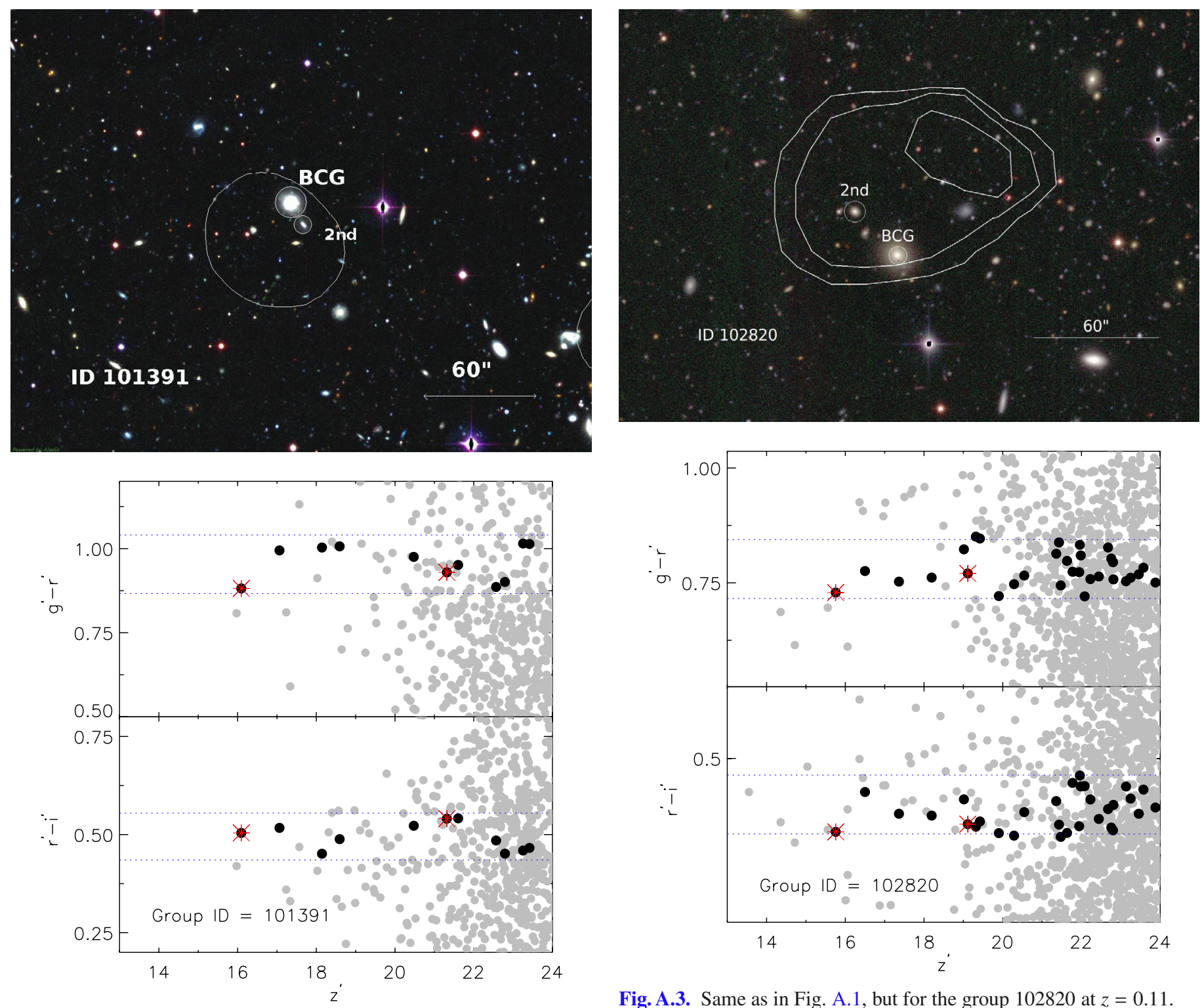

Fig. A.3. Same as in Fig. A.1, but for the group 102820 at $z=0.11$.

Fig. A.2. Same as in Fig. A.1, but for the group 101391 at $z=0.07$. 
G. Gozaliasl et al.: Evolution of the luminosity gap in X-ray galaxy groups
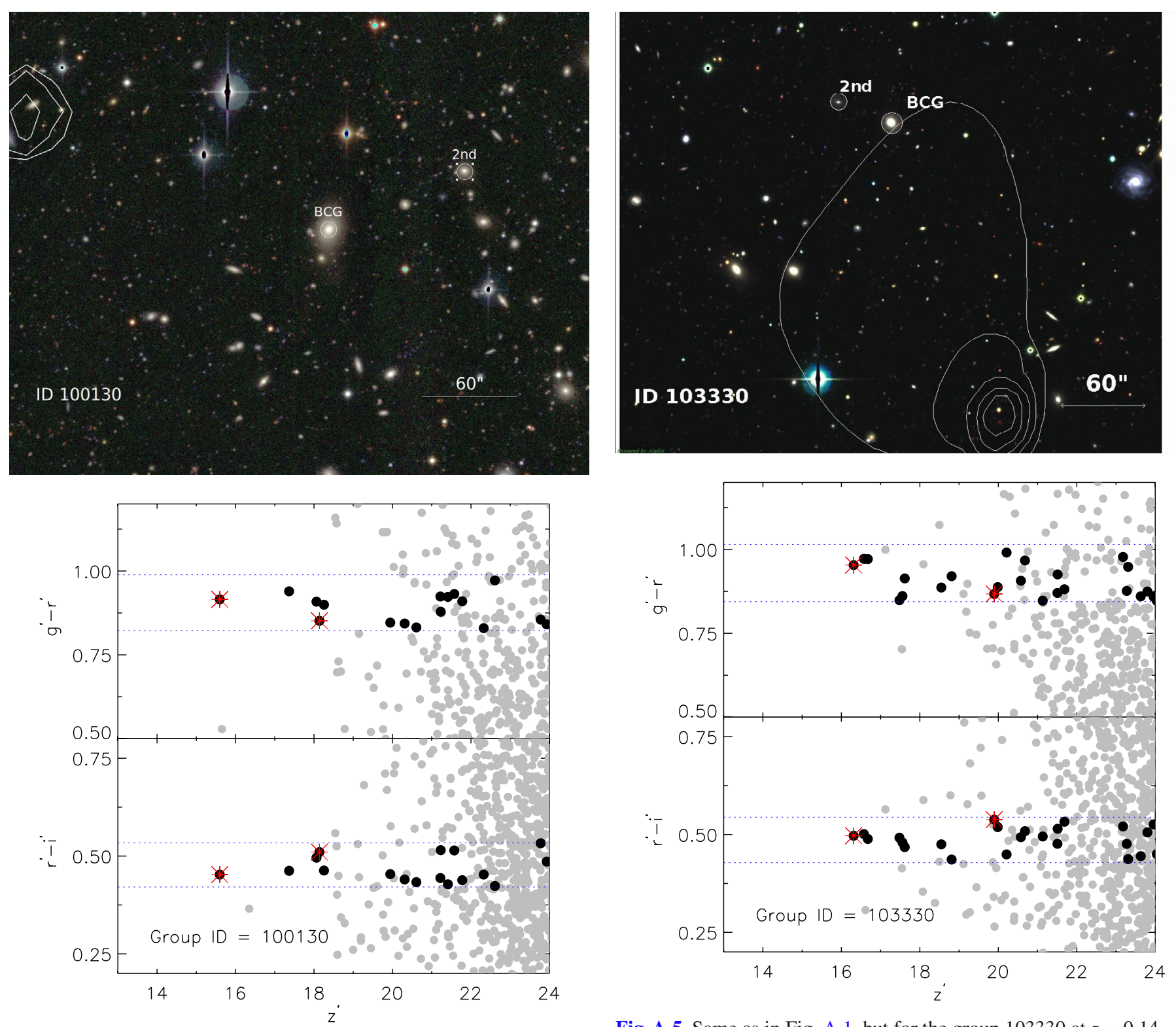

Fig. A.4. Same as in Fig. A.1, but for the group 100130, at $z=0.13$.

Fig. A.5. Same as in Fig. A.1, but for the group 103330 at $z=0.14$. 

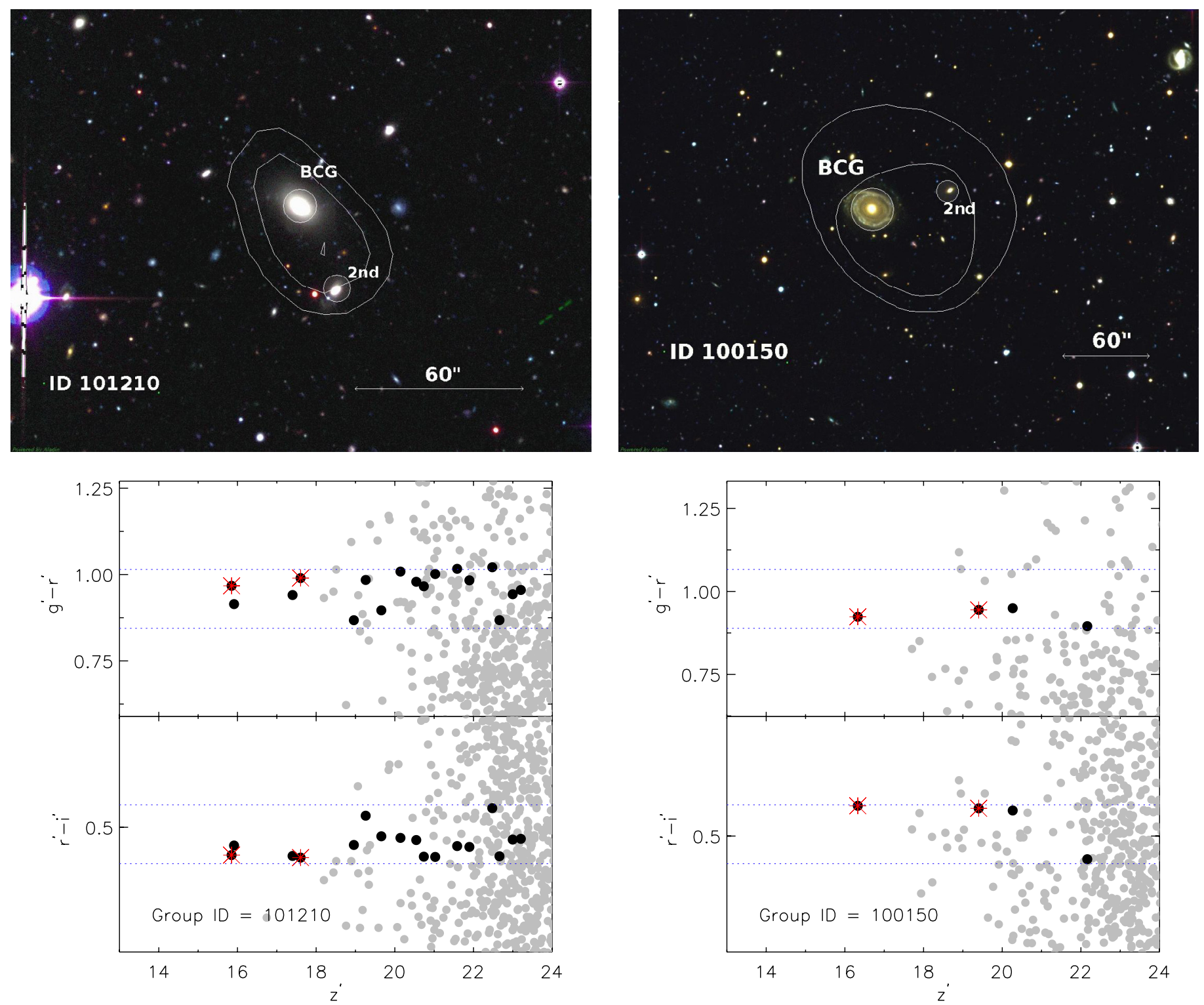

Fig. A.6. Same as in Fig. A.1, but for the group 101210 at $z=0.15$.

Fig. A.7. Same as in Fig. A.1, but for the group100150 at $z=0.16$. 
G. Gozaliasl et al.: Evolution of the luminosity gap in X-ray galaxy groups
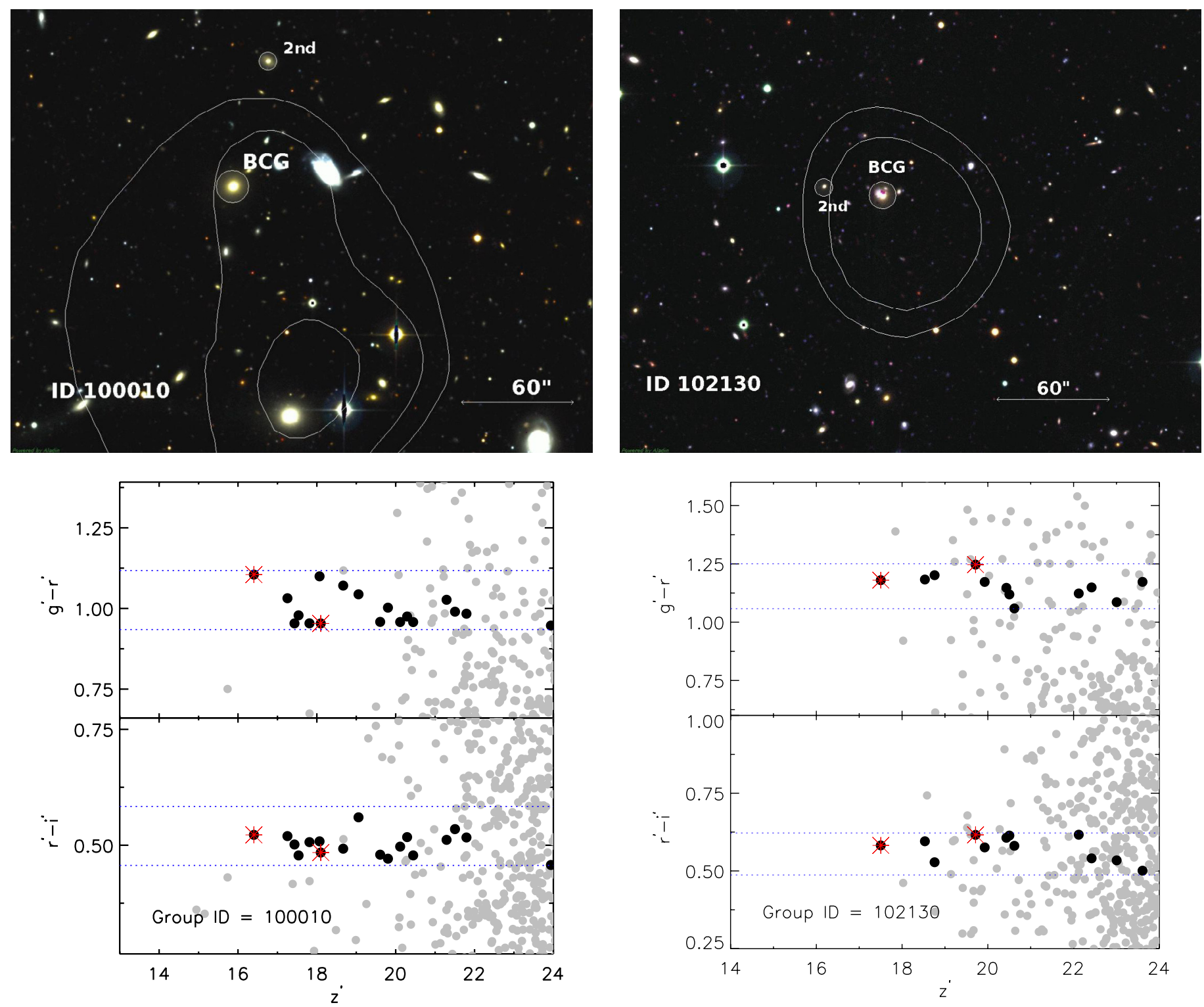

Fig. A.8. Same as in Fig. A.1, but for the group 100010, at $z=0.22$.

Fig. A.9. Same as in Fig. A.1, but for the group 102130 at $z=0.23$. 

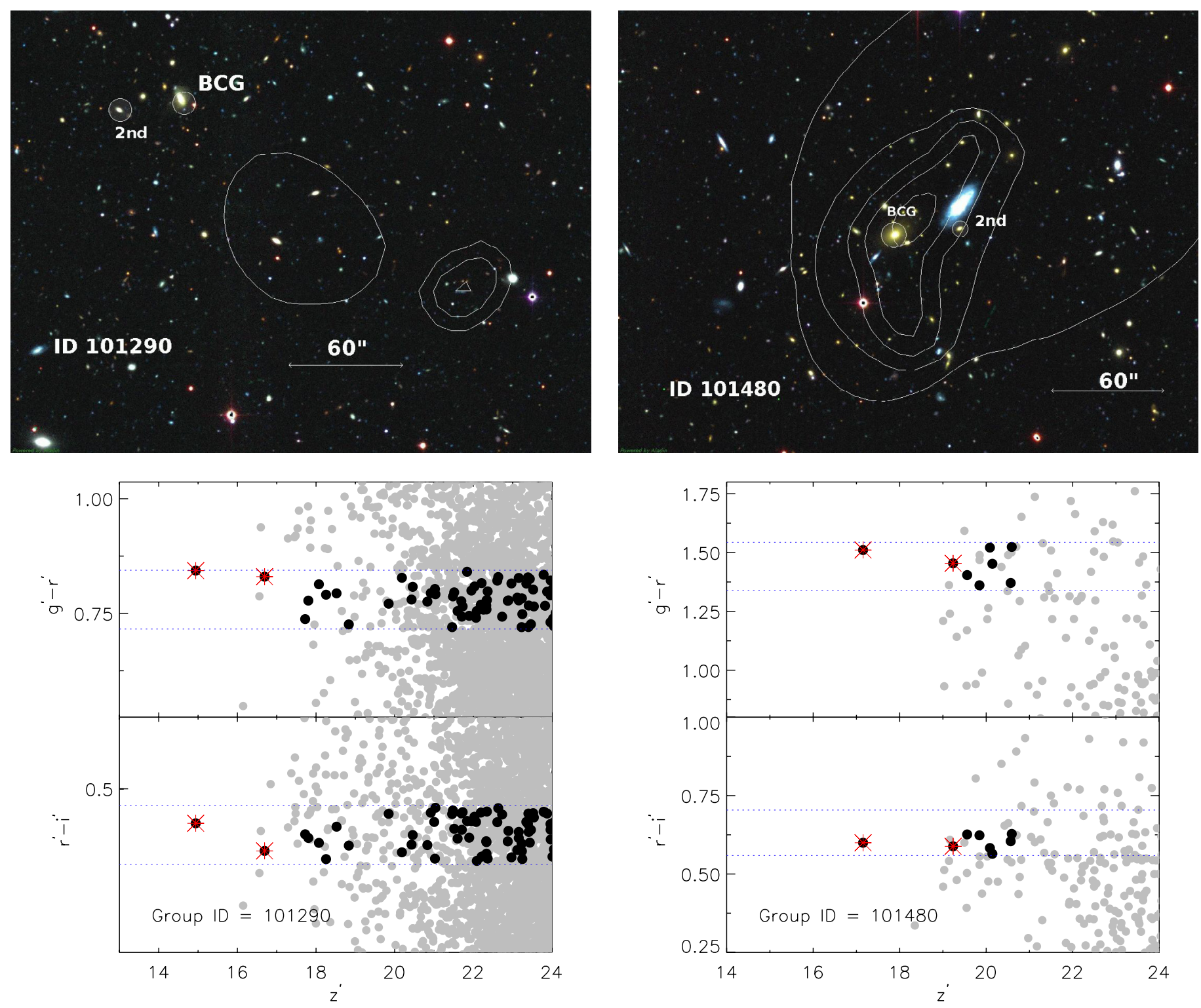

Fig. A.10. Same as in Fig. A.1, but for the group 101290 at $z=0.25$.

Fig. A.11. Same as in Fig. A.1, but for the group 101480, at $z=0.34$. 
G. Gozaliasl et al.: Evolution of the luminosity gap in X-ray galaxy groups
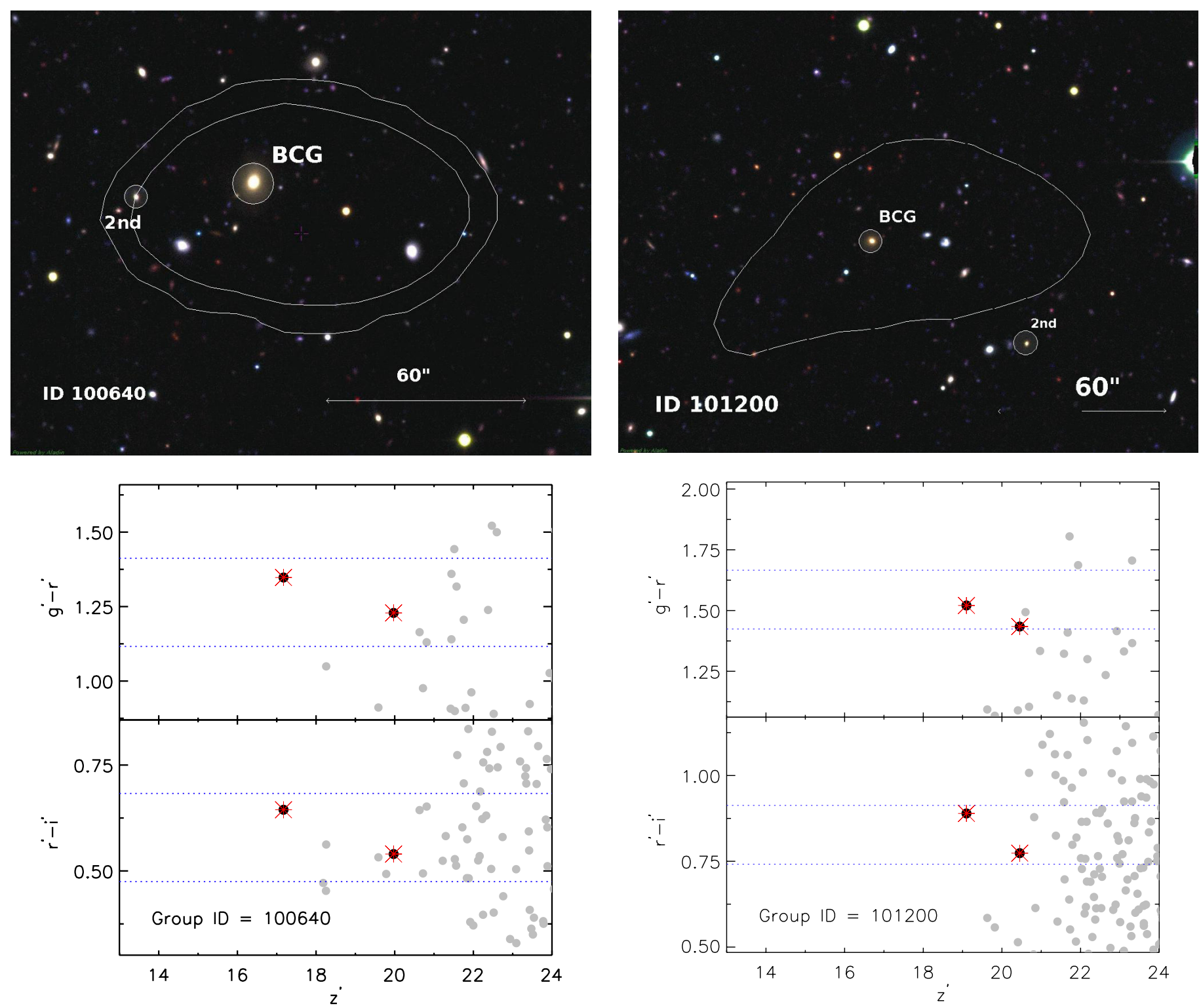

Fig. A.12. Same as in Fig. A.1, but for the group 100640, at $z=0.34$.

Fig. A.13. Same as in Fig. A.1, but for the group 101200 at $z=0.47$. The 2 nd brightest galaxy cannot be found inside $0.5 R_{200}$ due to completeness effect and the galaxy marked is at a $0.6 R_{200}$ distance to the center. 
A\&A 566, A140 (2014)
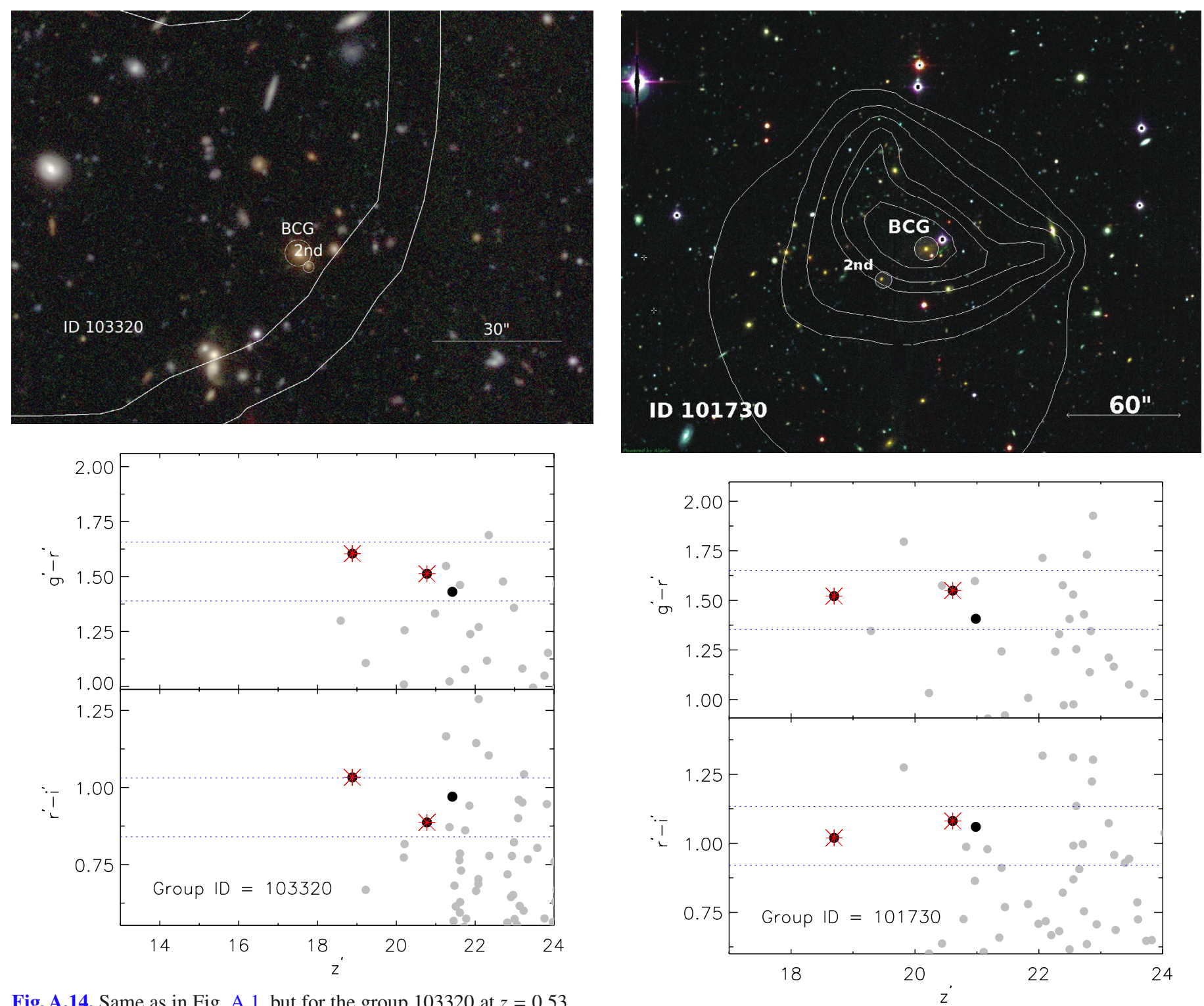

Fig. A.14. Same as in Fig. A.1, but for the group 103320 at $z=0.53$.

Fig. A.15. Same as in Fig. A.1, but for the group 101730, at $z=0.60$. 
G. Gozaliasl et al.: Evolution of the luminosity gap in X-ray galaxy groups
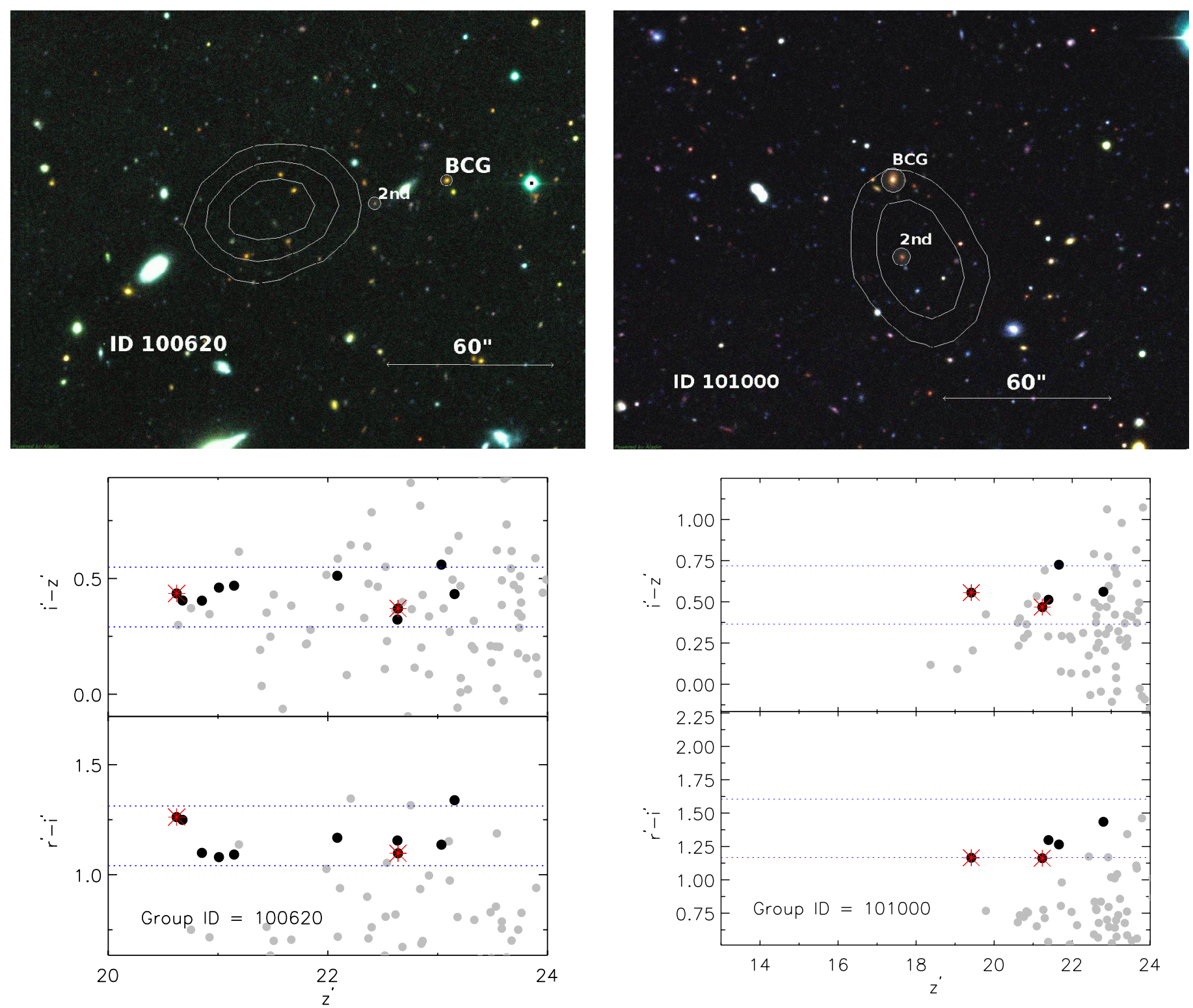

Fig. A.16. Upper panel: contours of extended X-ray emission overlaid on the CFHTLS RGB image of the fossil group 100620 at $z=0.66$. Middle panel: $i^{\prime}-z^{\prime}$ versus $z^{\prime}$. Lower panel: $r^{\prime}-i^{\prime}$ versus $z^{\prime}$. Filled black circles illustrate group members selected by the method described in Sect. 4.1. The BGG and second brightest satellite galaxy within $0.5 R_{200}$ have been marked with red asterisks within each color magnitude diagram. The upper and lower limits of colors have been shown by horizontal dotted blue lines.

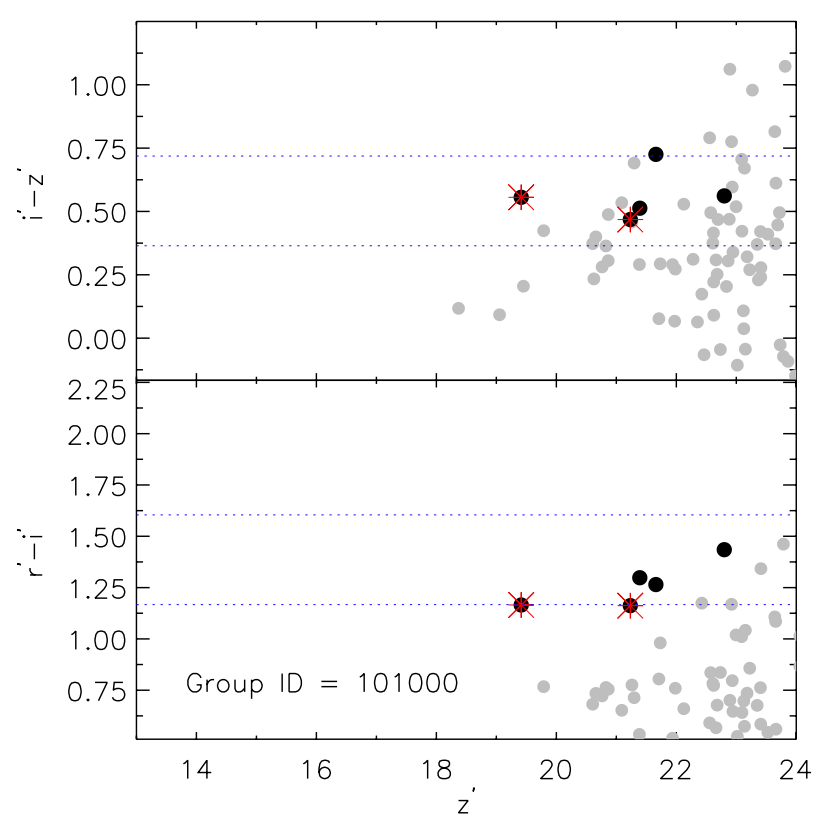

Fig. A.17. Same as in Fig. A.16, but for the group 101000 at $z=0.76$. 
A\&A 566, A140 (2014)
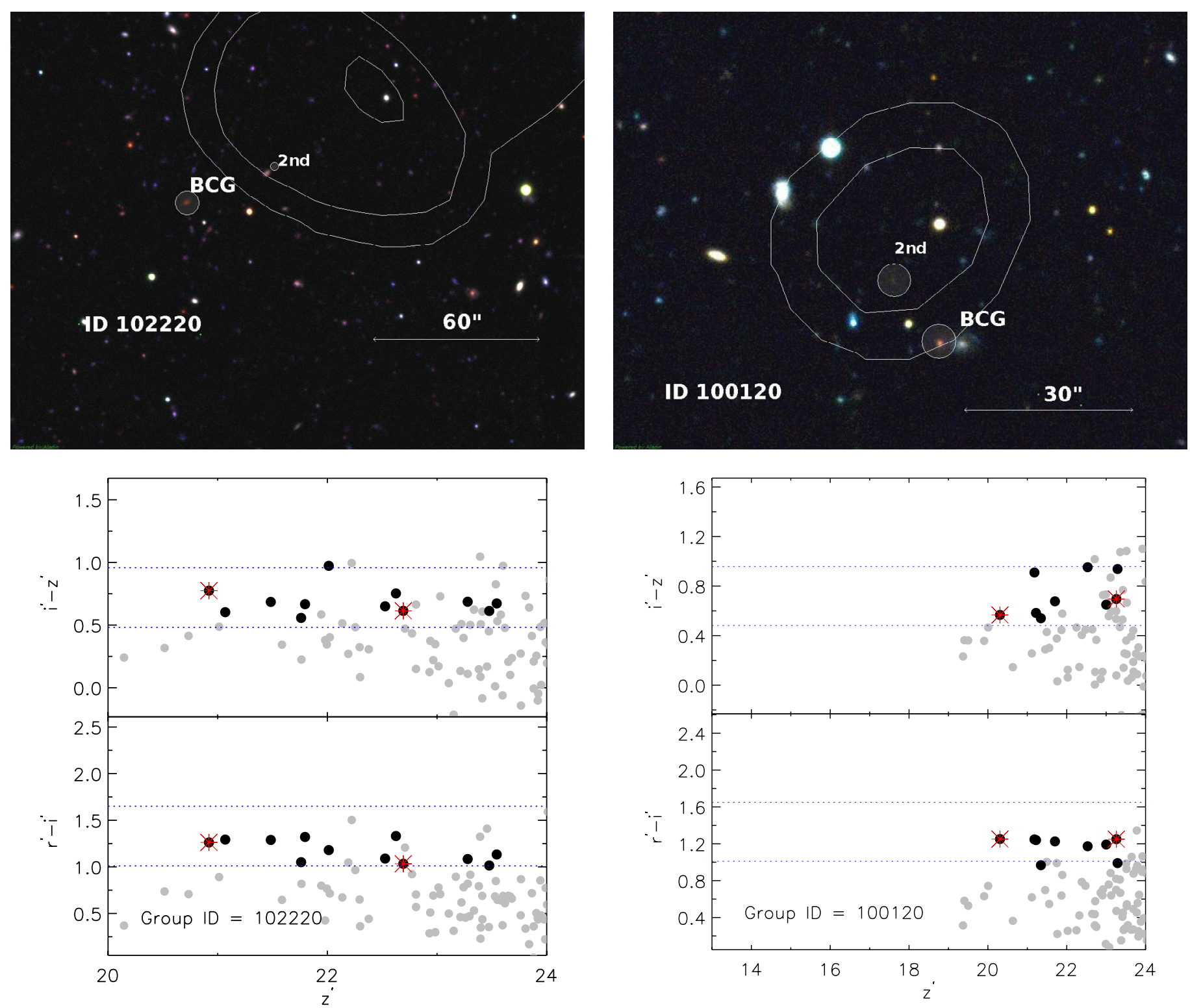

Fig. A.18. Same as in Fig. A.16, but for the group 101120 at $z=0.86$.

Fig. A.19. Same as in Fig. A.16, but for the group 100120 at $z=0.88$. 
G. Gozaliasl et al.: Evolution of the luminosity gap in X-ray galaxy groups
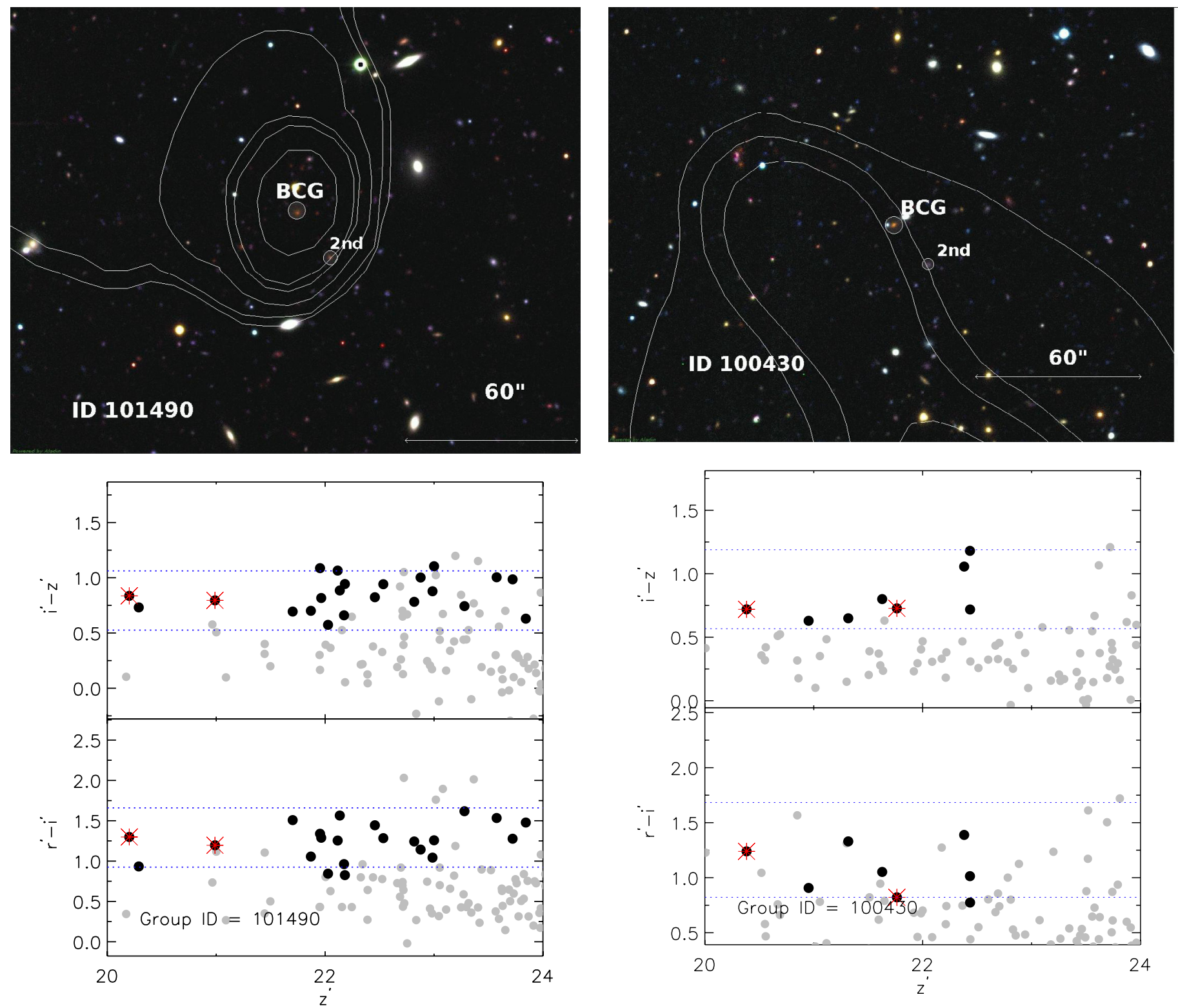

Fig. A.20. Same as in Fig. A.16, but for the group 101490, at $z=0.94$.

Fig. A.21. Same as in Fig. A.16, but for the group 100430, at $z=0.98$. 

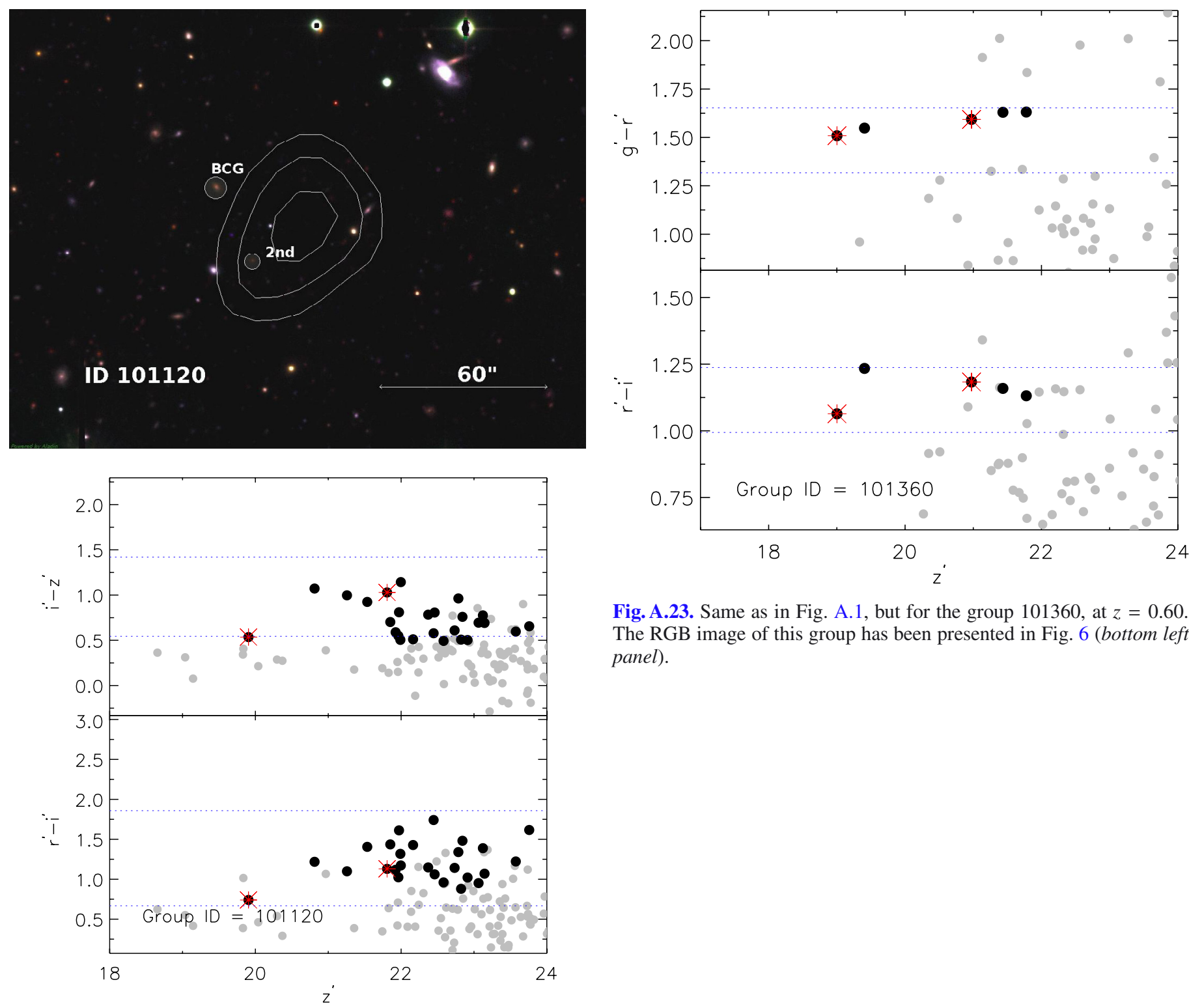

Fig. A.23. Same as in Fig. A.1, but for the group 101360, at $z=0.60$. The RGB image of this group has been presented in Fig. 6 (bottom left panel).

Fig. A.22. Same as in Fig. A.16, but for the group 101120, at $z=1.1$. 